\title{
GaBoDS: The Garching-Bonn Deep Survey
}

\section{Data release of the ESO Deep-Public-Survey ${ }^{\star, \star \star}$}

\author{
H. Hildebrandt ${ }^{1}$, T. Erben ${ }^{1}$, J. P. Dietrich ${ }^{1}$, O. Cordes $^{1}$, L. Haberzettl ${ }^{2}$, M. Hetterscheidt ${ }^{1}$, M. Schirmer ${ }^{3}$, \\ O. Schmithuesen ${ }^{2}$, P. Schneider ${ }^{1}$, P. Simon ${ }^{1}$, and C. Trachternach ${ }^{2}$
}

1 Argelander-Institut für Astronomie, Universität Bonn ${ }^{\star \star \star}$, Auf dem Hügel 71, 53121 Bonn, Germany
e-mail: hendrik@astro. uni-bonn.de
2 Astronomisches Institut der Ruhr-Universität-Bochum, Universitätsstr. 150, 44780 Bochum, Germany
3 Isaac Newton Group of Telescopes, Apartado de correos 321, 38700 Santa Cruz de La Palma, Tenerife, Spain

Received 29 September 2005 / Accepted 2 March 2006

\begin{abstract}
Aims. In this paper the optical data of the ESO Deep-Public-Survey observed with the Wide Field Imager and reduced with the THELI pipeline are described.

Methods. Here we present 63 fully reduced and stacked images. The astrometric and photometric calibrations are discussed and the properties of the images are compared to images released by the ESO Imaging Survey team covering a subset of our data.

Results. These images are publicly released to the community. Our main scientific goals with this survey are to study the high-redshift universe by optically pre-selecting high-redshift objects from imaging data and to use VLT instruments for follow-up spectroscopy as well as weak lensing applications.
\end{abstract}

Key words. surveys - galaxies: photometry - galaxies: high-redshift

\section{Introduction}

The ESO Deep-Public-Survey (DPS) is a multi-colour imaging survey carried out by the ESO Imaging Survey (EIS) team under the program IDs 164.0-0561 and 169.A-0725. It consists of optical data in the UBVRI-bands observed with the Wide Field Imager (WFI) at the $2.2 \mathrm{~m}$ telescope at La Silla and infrared data in the $J$ - and $K$ s-bands observed with SOFI at the New Technology Telescope. In this paper we present 63 reduced, photometrically and astrometrically fully calibrated, and stacked images of the optical part which are released to the scientific community ${ }^{1}$. These images were reduced and calibrated with our THELI reduction pipeline (Erben et al. 2005).

The main scientific driver for the ESO DPS were searches for high-redshift galaxies, distant clusters, high-redshift QSOs, low surface-brightness galaxies, and gravitational lensing studies. The survey was designed in a way to deliver a unique dataset also for studies on Galactic structure, very low-metallicity stars, white dwarfs, M-dwarfs, and field brown dwarfs. The deep imaging data can be used to pre-select objects by colour for follow-up spectroscopy with VLT instruments.

* Based on observations made with the European Southern Observatory telescopes obtained from the ESO/ST-ECF Science Archive Facility.

$\star \star$ Table 4 and appendices are only available in electronic form at http://www.edpsciences.org

$\star \star \star$ Founded by merging of the Sternwarte, Radioastronomisches Institut and Institut für Astrophysik und Extraterrestrische Forschung der Universität Bonn.

1 The images can be downloaded at http://marvin.astro.unibonn.de/DPS/ and are available via the ESO archive

http://archive.eso.org/archive/eso_data_products.html
It was intended to cover an area of three square degrees in three well separated regions of one square degree each at high galactic latitude called Deep1, Deep2, and Deep3. Each region consists of four adjacent WFI pointings $\left(34^{\prime} \times 33^{\prime}\right)$ at the same declination named $a, b, c, d$ in order of decreasing right ascension. Table 1 summarises the positions of the twelve DPS fields. The Deep1 region was chosen to overlap with the ATESP radio survey (Prandoni et al. 2000). The Chandra Deep Field South (CDFS) is included in the Deep2 region (centred on the field Deep2c), and Deep3 is a random, empty, high galactic latitude field positioned in such a way that DPS observations are possible over the whole calendar year.

Our group has developed a wide-field imaging reduction pipeline and decided to reduce the DPS data in late 2003 as an ideal test case of a unique, large dataset fitting our scientific goals. We are mainly interested in searches for Lyman-break galaxies (LBGs) and weak-lensing studies supported by photometric redshifts. In November 2004 the EIS team released 40 reduced images of the optical part of the DPS which enabled us to compare the performance of our pipeline to a different one. The released EIS data cover a subset of our data release and are available via the ESO archive.

The paper is organised as follows. In Sect. 2 the instrument, its photometric system, the observing strategy, and the raw data are described. Section 3 gives a short summary of the data reduction with the THELI pipeline with emphasis on steps which are important for the end user. The properties of our released images are presented in Sect. 4, and in Sect. 5 these properties are compared to the EIS data release of the ESO DPS. A summary of the data release and an outlook on projects carried out with the DPS data is given in Sect. 6 . 
Table 1. Positions and available colours of the twelve DPS fields and the two mispointings (Deeple and Deep1f; see text). The region Deep1 overlaps with the ATESP radio survey (Prandoni et al. 2000) and the field Deep2c is centred on the Chandra Deep Field South.

\begin{tabular}{rccc}
\hline \hline Field & $\begin{array}{c}\text { RA [h m s] } \\
\text { J2000.0 }\end{array}$ & $\begin{array}{c}\text { Dec [d m s] } \\
\text { J2000.0 }\end{array}$ & $\begin{array}{c}\text { Avail. } \\
\text { colours }\end{array}$ \\
\hline Deep1a & $22: 55: 00.0$ & $-40: 13: 00$ & $U B V R I$ \\
$\mathrm{~b}$ & $22: 52: 07.1$ & $-40: 13: 00$ & $U B V R I$ \\
$\mathrm{c}$ & $22: 49: 14.3$ & $-40: 13: 00$ & $V R I$ \\
$\mathrm{~d}$ & $22: 46: 21.4$ & $-40: 13: 00$ & - \\
Deep2a & $03: 37: 27.5$ & $-27: 48: 46$ & $R$ \\
$\mathrm{~b}$ & $03: 34: 58.2$ & $-27: 48: 46$ & $U B V R I$ \\
$\mathrm{c}$ & $03: 32: 29.0$ & $-27: 48: 46$ & $U B V R I$ \\
$\mathrm{~d}$ & $03: 29: 59.8$ & $-27: 48: 46$ & $R$ \\
Deep3a & $11: 24: 50.0$ & $-21: 42: 00$ & $U B V R I$ \\
$\mathrm{~b}$ & $11: 22: 27.9$ & $-21: 42: 00$ & $U B V R I$ \\
$\mathrm{c}$ & $11: 20: 05.9$ & $-21: 42: 00$ & $U B V R I$ \\
$\mathrm{~d}$ & $11: 17: 43.8$ & $-21: 42: 00$ & $B V R I$ \\
\hline Deep1e & $22: 47: 47.9$ & $-39: 31: 06$ & $U R I$ \\
$\mathrm{f}$ & $22: 44: 58.4$ & $-39: 31: 54$ & $I$ \\
\hline
\end{tabular}

Table 2. Characteristics of the filters used for the DPS. For the computation of the effective wavelength and the filter's width the CCD efficiency is included.

\begin{tabular}{|c|c|c|c|c|}
\hline Filter & $\overline{\mathrm{ESO} \#}$ & eff. WL $[\AA]$ & 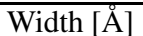 & AB corr. $[\mathrm{mag}]$ \\
\hline$U 35060$ & 877 & 3429 & 705 & 1.1 \\
\hline$U_{-} 38$ & 841 & 3647 & 373 & 0.8 \\
\hline$B$ & 842 & 4589 & 871 & -0.1 \\
\hline V & 843 & 5377 & 793 & 0.0 \\
\hline$R$ & 844 & 6504 & 1502 & 0.2 \\
\hline I & 845 & 8635 & 1425 & 0.5 \\
\hline$I \_E I S$ & 879 & 8057 & 1402 & 0.5 \\
\hline
\end{tabular}

\section{The raw data}

All optical data of the DPS were observed with the Wide Field Imager (WFI). This multi-chip, focal-reducer CCD camera has a field of view of $34^{\prime} \times 33^{\prime}$ with a filling factor of $\sim 96 \%$. Seven broad-band filters are used for the DPS some of which are very different from the standard Johnson-Cousins UBVRI filters. Their properties are summarised in Table 2 . The $U$-band filter ESO \# 841 and the I-band filter ESO \# 845 were replaced by new filters for DPS observations after some time. In Table 3 the scheduled exposure times and expected limiting magnitudes are shown which were chosen to suffice the scientific goals as described above. For our reduction of the DPS we used all the raw data available until December 2005 including images from the two large programmes 164.O-0561 and 169.A-0725 (P.I. Krautter for both) and, moreover, data from the programmes 67.A-0244 (P.I. Schneider) and 75.A-0280 (P.I. Hildebrandt). For the field Deep2c we added also data from the programmes 168.A-0485 (P.I. Cesarsky), 64.H-0390 (P.I. Patat), 66.A-0413 (P.I. Clocchiatti), 68.D-0273 (P.I. Cappellaro), 68.A-0443 (P.I. Clocchiatti), 70.A-0384 (P.I. Vanzi), and 74.A-9001 (P.I. Kuijken), and we used the WFI commissioning data and data from the COMBO17 survey (P.I. Meisenheimer) described in Wolf et al. (2004).

Unfortunately, the optical observations of the DPS were not finished. Not all fields were observed to the scheduled depths in all filters. Furthermore, some observations were executed under bad sky conditions so that these images are excluded from the data reduction. There are also some mispointings present in the DPS raw data for which the FITS header contains the
Table 3. Scheduled exposure times and expected limiting magnitudes for the images in the different filters. The limiting magnitudes correspond to $5 \sigma$ sky level in an aperture of $2^{\prime \prime}$.

\begin{tabular}{lrc}
\hline \hline Filter & Exp. time [s] & Mag lim. [Vega mag] \\
\hline$U \_35060$ & 43200 & 25.7 \\
$U \_38$ & 61200 & 26.0 \\
$B$ & 12600 & 26.1 \\
$V$ & 9000 & 26.0 \\
$R$ & 9000 & 26.1 \\
$I$ & 27000 & 25.5 \\
$I \_E I S$ & 27000 & 25.5 \\
\hline
\end{tabular}

correct coordinates of one DPS field while the actually observed area is offset by some arcminutes. Some fields (especially the field Deep2c centred on the Chandra Deep Field South) were observed by different programmes to much greater depth, and we also included these data in our reduction. The estimates of the limiting magnitudes in Table 3 were quite optimistically calculated at a time when the WFI did not yet exist. The final images are significantly shallower (see Table 4). For all these reasons the DPS is rather heterogeneously deep, and this fact should be kept in mind when dealing with data from different DPS fields.

The desired depth usually requires observations in different nights for every field in each colour. For many fields, observations spread over more than a year and images taken under very different photometric conditions have to be combined. Even the instrument setup (baffling, camera rotation etc.) changed over the time of DPS observations. Furthermore, to reach a final coadded image with an exposure time as uniform as possible over the field and in order to get good relative astrometry and photometry between the chips, a wide dither pattern was chosen for the DPS. The whole observing strategy (dither pattern, exposure times of the individual images etc.) was adapted and optimised over the years of DPS observations.

\section{Data reduction}

The data reduction was performed with our THELI pipeline described in detail in Erben et al. (2005). All the raw data were requested from the ESO Science Archive Facility.

\subsection{Pre-reduction}

The data were sorted in so-called observation runs which include science, calibration, and standard star images from some adjacent nights. In the $U B V R$-bands an observation run can easily contain data from one $(R)$ or even two weeks $(U B V)$. The strong fringe patterns in the $I$-band are variable from night to night and even within one night, so that $I$-band runs usually contain data from only one or two hours of observation.

On this run basis, the science images were pre-reduced which includes overscan correction, bias subtraction, flatfielding, super-flatting, and in the $R$ - and $I$-band also fringeremoval. No correction for the inhomogeneous illumination (see Koch et al. 2004) was applied. Weight images were created containing flags for bad pixels, bad columns, and other image defects like satellite tracks which were masked out by hand. The Landolt standard star images which are available for most nights were reduced in the same way as the science images. 


\subsection{Absolute photometric calibration}

Here we describe in a bit more detail the absolute photometric calibration which is not covered in Erben et al. (2005). All objects from the standard star frames were extracted and matched to a photometric standard star catalogue, the Landolt catalogue (Landolt 1992) in the $U$-band and the Stetson catalogue (Stetson $2000)^{2}$ for the other bands, respectively. Per WFI field, this usually led to more than a thousand matched stars per night in the $B V R I$-bands and to around a hundred in the $U$-band which could be used for calibration. The observed instrumental WFI magnitudes, $m_{\text {inst. }}$, were related to the standard Johnson-Cousins system, $m_{\mathrm{JC}}$, by the following equation:

$m_{\mathrm{JC}}=\mathrm{ZP}+m_{\mathrm{inst} .}+$ Colour $\cdot \mathrm{CT}+$ Airmass $\cdot \mathrm{EXT}$,

with ZP the photometric zeropoint, CT the colour term, and EXT the extinction coefficient. It should be stressed that the photometric system of WFI is very different from the standard system and this linear relation fails for some filters (especially the $U$ - and $B$-filters) and objects with large colour terms. It is in general necessary to work with instrumental magnitudes when doing photometry with WFI. Depending on the number of matched standard stars, different solutions were chosen for the different nights. In nights with standard star fields spanning over a wide range of airmasses, the instrumental magnitudes could be fit to the standard system's magnitudes with a three-parameter fit using the zeropoint, colour term, and extinction coefficient as free parameters (for EXT only negative values are fitted). For nights with poorer coverage in airmass, the extinction term was fixed to a default value (taken from the WFI website, http://www.ls.eso.org/lasilla/ sciops/2p2/E2p2M/WFI/) and a two-parameter fit with the zeropoint and colour term as free parameters was applied. Sometimes, especially in the $U$-band, also colour coverage was not good enough to fit for the colour term. Then only the zeropoint is estimated in a one-parameter fit with the colour term also set to a default value. The decision was taken with the help of plots showing the differences between instrumental magnitude and standard star magnitude plotted versus airmass and versus colour (see Fig. 1 for an example). The choice from these plots should be regarded as the first run of our absolute photometric calibration. Experience shows that it is often not possible to entirely judge the photometric quality of a whole night from these plots alone. Sometimes photometric conditions change over the night which cannot be detected from these standard star exposures typically observed two or three times a night only. Thus, we evaluate the calibration by three further means, namely corrected photometric zeropoints (see Sect. 3.4), colour-colour-diagrams of stars (see Fig. 3), and apparent magnitude number-counts (see Fig. 4). The method based on corrected photometric zeropoints fully exploits the long term characteristic of the DPS with observations in one field and colour usually spanning many nights. All photometric solutions were added to the image header so that later changes could be made (e.g. from 2-parameter fit to 1-parameter fit). Every night was given a unique socalled GaBoDS ID starting with 1 on January, 1st, 1999. The photometric solutions for all the nights of the DPS can be found in the Appendix (see Tables A.1 to A.7). On December, 13, 2004 the EIS team also published their photometric

2 Available at http://cadcwww.hia.nrc.ca/cadcbin/ wdbi.cgi/astrocat/stetson/query solutions for all nights calibrated by them which can be found on the $w_{e b}^{3}$.

\subsection{Transition from run to set}

For every run websites were created containing essential image information like exposure time, seeing, and other important quantities ${ }^{4}$. The further steps like relative astrometric and photometric calibration and coaddition require all images from the same coordinates. Hence the images were re-distributed from the runs into so-called sets containing pre-reduced images from different epochs but at the same sky position.

\subsection{Astrometric and photometric calibration}

The astrometric calibration was performed with ASTROMETRIX (Radovich 2002) on catalogues created with SExtractor (Bertin $\&$ Arnouts 1996). The external astrometry was fixed with respect to the USNO-A2.0 (Monet et al. 1998) catalogue. LDAC tools were used to do the relative photometric calibration and to bring all images to the same flux scale. First, the relative zeropoints $\mathrm{ZP}_{\text {rel }}$ of each single chip was estimated from the flux differences of overlap objects. At this stage it was required that the sum of the relative zeropoints of all images of the set equals zero, $\sum_{i} \mathrm{ZP}_{\mathrm{rel}, i}=0$. Then the images from calibrated nights (see Sect. 3.2) were taken and so-called corrected zeropoints $\mathrm{ZP}_{\text {corr }}$ were calculated in the following way:

$\mathrm{ZP}_{\text {corr }, i}=\mathrm{ZP}+$ Airmass $\cdot \mathrm{EXT}+\mathrm{ZP}_{\text {rel }, i}$,

with ZP and EXT being the zeropoint and the extinction coefficient of the particular night, respectively. Theoretically, these corrected zeropoints should then coincide for photometric frames, and usually this assumption holds for most sets to within $\sim 0.05$ mag. Deviations were used as hints for bad absolute photometric calibrations or changing photometric conditions over the night. That is to say, if conditions change from the standard star exposure to the scientific exposure over the course of the night, this can be detected from the corrected zeropoints. Furthermore, it can happen that from the plots shown in Fig. 1 it is difficult to decide whether solution number two or three should be chosen. Sometimes it is even not entirely clear whether a night should be rejected completely for the absolute photometric calibration. At this point the distribution of the corrected zeropoints can help to identify nights where obviously a bad decision was taken. In Fig. 2 such a situation is illustrated by an extreme example. There, the solutions chosen in the first run of the absolute photometric calibration yielded a large scatter in the corrected zeropoints. After rejecting some nights with small numbers of standard stars or zeropoints far from the default values (hints for non-photometric conditions) in the second run, the scatter in the corrected zeropoints decreased considerably and the mean of this distribution was taken as the zeropoint for the coadded image. After such a treatment the distribution of the corrected photometric zeropoints shows a HWHM scatter of $\sigma \lesssim 0.05$ mag for all images.

With the current calibration plan for WFI, taking standard star frames mostly at the beginning and the end of the night (sometimes in twilight), it is not possible to account for changing photometric conditions during one night. Thus, a night has to be accepted or rejected and no discrimination between good

\footnotetext{
3 http://www.eso.org/science/eis/surveys/ release_70000027_Photometry.html

4 Available at: http://marvin.astro.uni-bonn.de/DPS/
} 
$R$-band, night: 2000-08-25
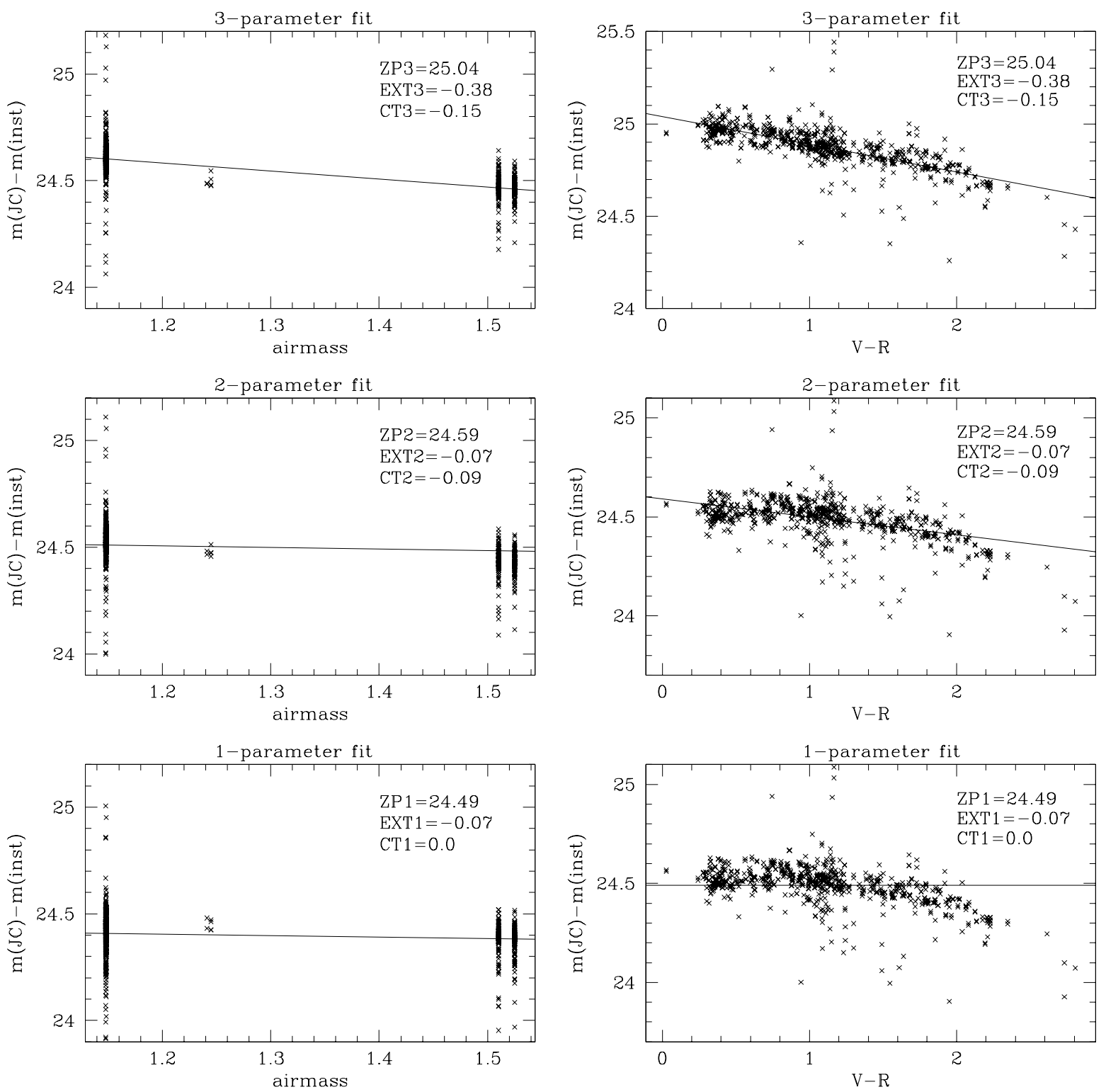

Fig. 1. Plot for the decision between photometric solutions of one night. The first row shows the results for the three-parameter fit, the difference between instrumental magnitude (data from all eight chips) and standard star magnitude plotted versus airmass (for colour zero) on the left and versus colour (for airmass one) on the right. In the second and third row the same diagrams are shown for the two- and the one-parameter fit. For this particular night, the three-parameter fit yields an extinction coefficient that is much too large (compared to the default value EXT $=-0.07$ ) resulting in a bad zeropoint. Obviously this night is not totally photometric. Nevertheless, experience shows that such nights can often be still used to estimate a zeropoint and sometimes even a colour term for the images. The one-parameter fit was chosen here because the non-linearities visible in the colour plots for the one- and the two-parameter fits influence the value for CT2, resulting again in a zeropoint ZP2 that is too large. For ideal photometric nights the values from the three different fits show only a very small scatter. The instrumental photometric errors are not shown in these plots since at the bright magnitudes of the Stetson/Landolt standard stars they are negligible and would hardly be visible.

and bad data from one night is feasible since the scientific data usually span the whole night.

After the absolute photometric calibration, the fluxes of the images were multiplied by a factor $10^{-0.4 Z P_{\text {rel, }}} / t_{i}$, with $t_{i}$ being the exposure time of the single image. Thus, the counts in our final coadded image are scaled to one second.

\subsection{Coaddition}

The calibrated images then entered the coaddition which was performed with SWarp (Bertin 2003). We chose a weighted mean coaddition to maximise the $S / N$ of our final images relying on the efficiency of our weight maps. Some quantities like seeing, sky background, mean stellar ellipticities etc. were estimated for every single frame during the relative astrometric and photometric calibration step. By these values, single frames could be excluded from the coaddition to avoid a degradation of the final image. In fact, different coaddition conditions were often chosen so that for some fields there is more than one image available in some filters. For example, it can be useful for certain scientific purposes to have an image as deep as possible (e.g. for deep multicolour photometry) while seeing is not so important, whereas for other scientific applications a very good seeing 


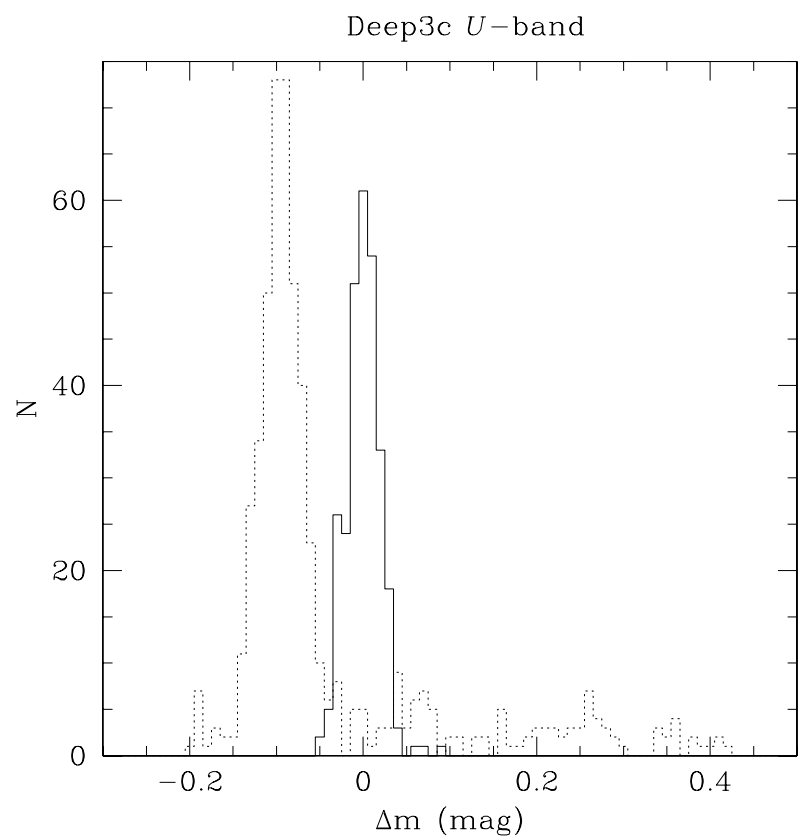

Fig. 2. Distribution of the differences $\Delta m$ between the mean of the corrected zeropoints $\frac{1}{N} \sum_{i} \mathrm{ZP}_{\text {corr }, i}$ and the individual corrected zeropoints $\mathrm{ZP}_{\text {corr } i}$ for the field Deep3c ( $U$-band). The dashed line represents the distribution before selecting different photometric solutions by hand, and the solid line represents the distribution after this selection.

is mandatory (e.g. for weak lensing studies). The images were given a unique coaddition ID and the selection criteria applied to the single frames entering the coaddition were written to the FITS header (see Table C.1).

\section{Released data}

\subsection{Images}

During the reduction of the DPS we produced 63 coadded images and their corresponding weight maps. The basic properties of the coadded images are summarised in Table 4. Usually the first coaddition aimed at including as many single exposures as possible to maximise the total exposure time. Exposures with a seeing of $>2^{\prime \prime}$, unusual high background fluxes, or large relative photometric zeropoint offsets compared to the rest of the set (indicative of twilight, moonlight, or clouds) were excluded from the coaddition. These coadded images were then assigned the letter "A" for "All". For example, the first coaddition of a Deep1c image then has the coaddition ID D1CA. If the exposures allowed for a second image with better seeing (but certainly with reduced exposure time) a second coaddition was performed and the letter "S" for "Seeing" was assigned to this image (e.g. coaddition ID D1BS). Sometimes, even further conditions were applied to the single frames for another coaddition then denoted by arbitrary letters like "C" or "G". During 2005 more data from the GOODS programme became available for the field Deep2c. Thus, further images denoted by a letter " $B$ " were created with the same conditions as the images with assigned letter "A" but including more data. Only these "D2CB" images are shown in Table 4.

The mispointings were processed, too. Therefore our data release contains two new fields called Deep1e and Deep1f that are not original DPS fields (see Table 1) but that are nevertheless quite deep in some bands and may be useful for some applications.

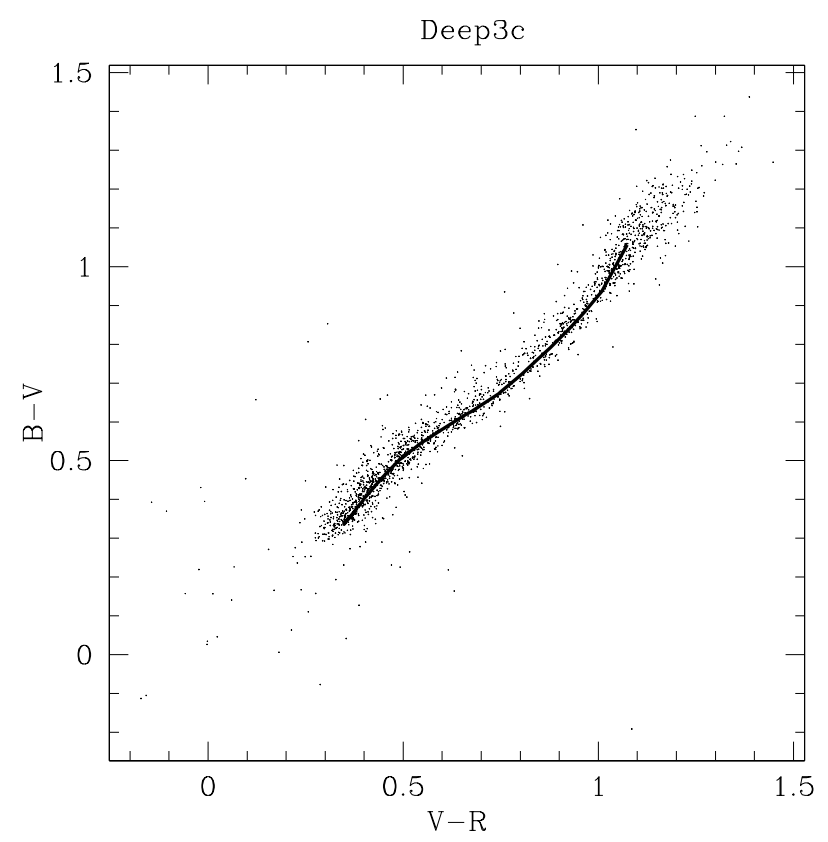

Fig. 3. Colour-colour diagram for stars in the field Deep3c in comparison to an isochrone from Girardi et al. (2002). The isochrone was calculated for stars with a metallicity of $Z=0.004(\approx 1 / 5 \times$ solar $)$ and an age of $10 \mathrm{Gyr}$. Only stars with an initial mass of $m_{\mathrm{ini}}<0.92 M_{\odot}$ were included.

A description of the GaBoDS image headers can be found in Appendix C.

\subsection{Photometric accuracy}

Since we did not perform an illumination correction in the prereduction step, a minimum photometric error of about 0.05 mag (see Koch et al. 2004) is present when using the same zeropoint over the whole field. In order to check the absolute photometric calibration, catalogues of all objects in the coadded images were created and the colours of stars (selected by the SExtractor-CLASS_STAR parameter) were plotted in colour-colour diagrams. These measured colours were compared to theoretical stellar isochrones by Girardi et al. (2002). An exemplary plot is shown in Fig. 3. After the thorough inspection of the corrected zeropoints no offsets larger than $\sim 0.15 \mathrm{mag}$ (perpendicular to the track) were present in these colour-colour diagrams.

Judging from these plots we estimate the error of our absolute photometric calibration to be below $\sim 0.1$ mag for our released images, in most cases better.

For an alternative check of the absolute photometric calibration, the number-counts of objects were compared to the object number-counts of our old, photometrically well-calibrated Chandra Deep Field South data (ESO Press Photos 02a-d/03) in $B V R$ and to the Chandra Deep Field South data from Arnouts et al. (2001) in $U \_35060, U \_38$, and $I$, respectively. An example is shown in Fig. 4. There is good agreement in these plots for all images within the expected field-to-field variance (especially at brighter magnitudes). For the $I \_E I S$ filter no such comparison was performed.

For the Chandra Deep Field South, an extensive database of spectroscopically observed objects from the VIMOS-VLTDeep-Survey (VVDS, Le Fèvre et al. 2004) exists. We compared the spectroscopic redshifts of all 407 VVDS galaxies with 


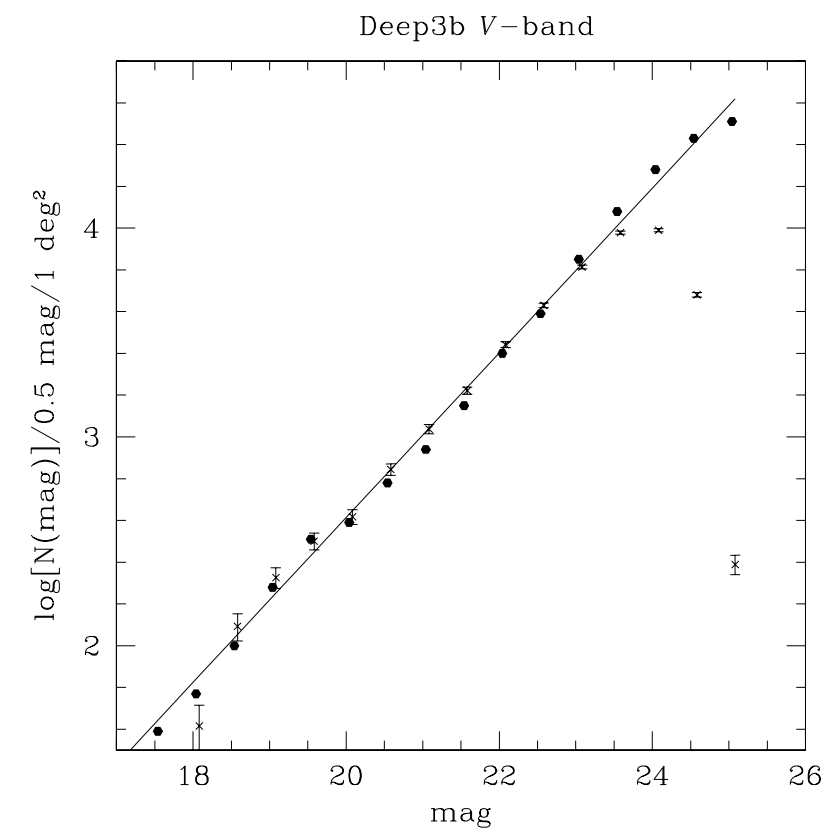

Fig. 4. Apparent $V$-band magnitude number-counts for objects in the field Deep3b (crosses) in comparison to the well-calibrated field Deep2c (hexagons) showing no significant offset.

$R<23$ and secure spectroscopic redshifts to photometric redshift estimates from the public photometric redshift code Hyperz (Bolzonella et al. 2000) based on our UBVRI photometry in the field Deep2c. Therefore we matched the seeing of the images in the different bands by convolving them with Gaussian kernels of the appropriate size. For the photometric redshift estimation we used the templates created from the synthetic spectral libraries of Bruzual \& Charlot (1993). The results are shown in Fig. 5. An outlier is defined as an object with $\left|z_{\text {spec }}-z_{\text {phot }}\right|>1$ and the dispersion is estimated via

$\sigma^{2}=\frac{\sum_{i=1}^{N}\left(z_{\mathrm{spec}, i}-z_{\mathrm{phot}, i}\right)^{2}}{N}$,

excluding outliers. These results are at least as good as the estimates from simulations given in Bolzonella et al. (2000) for the $U B V R I$ filter set, thus indicating a robust photometric calibration.

\subsection{Astrometric accuracy}

While overlap astrometry was used for all single chips entering the coaddition in one band, the final images of different bands were processed astrometrically independently and the catalogues produced for the colour-colour diagrams are also used to check the internal astrometric accuracy between the different colours. The positional differences of associated objects from the $B$ - and $V$-band images for the field Deep1b are plotted in Fig. 6. The dependence of these differences on chip position is shown in Fig. 7 where no residual effects like chip boundaries etc. are visible. For all fields, the distribution of the position differences between two bands are well described by a Gaussian with a half width at half maximum of $\sigma \lesssim 0.2$ (see Table 4). A slightly higher internal astrometric accuracy could have been obtained by using an astrometric catalogue from one band as the reference for the other bands. Given our already high accuracy of $\sigma<1$ pix this step was not performed for flexibility reasons. The quoted accuracy should suffice for almost all scientific goals.

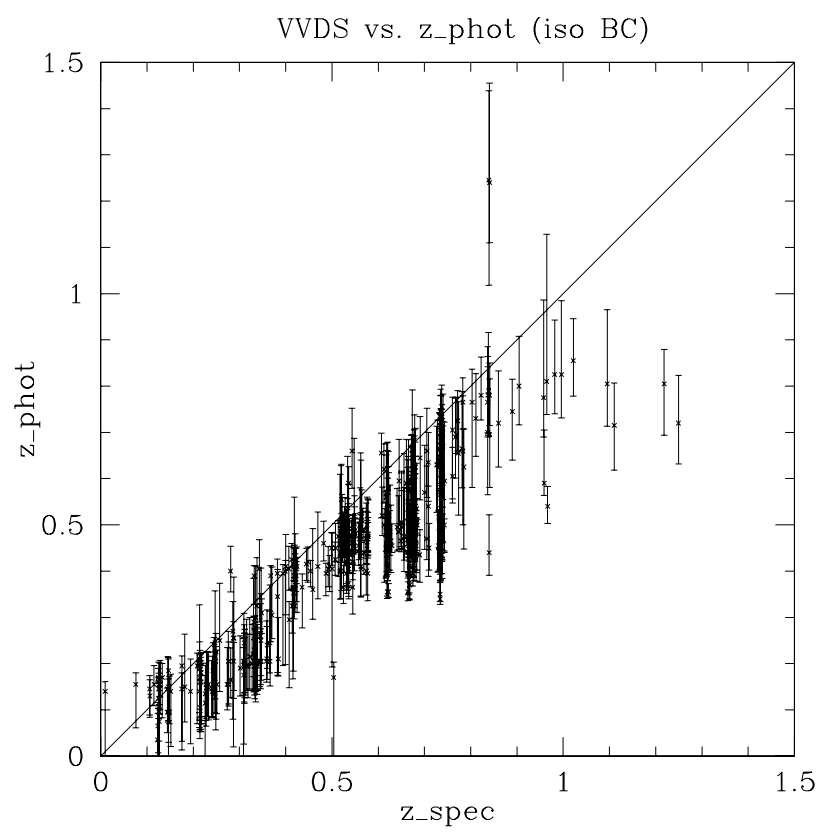

Fig. 5. Spectroscopic redshifts versus photometric redshifts for galaxies in the field Deep2c with $R<23$ from the VVDS (407 objects with secure spectroscopic redshifts and Hyperz-68\%-confidenceintervals smaller than $0.23 /(1+z))$. The errorbars represent the Hyperz-68\%-confidence-interval. The standard deviation and the outlier fraction (see text) are $\sigma=0.14$ and $l=0.25 \%$. The systematic underestimation of the redshifts for galaxies with $z<1$ is reported in Bolzonella et al. (2000) for the UBVRI filter set.

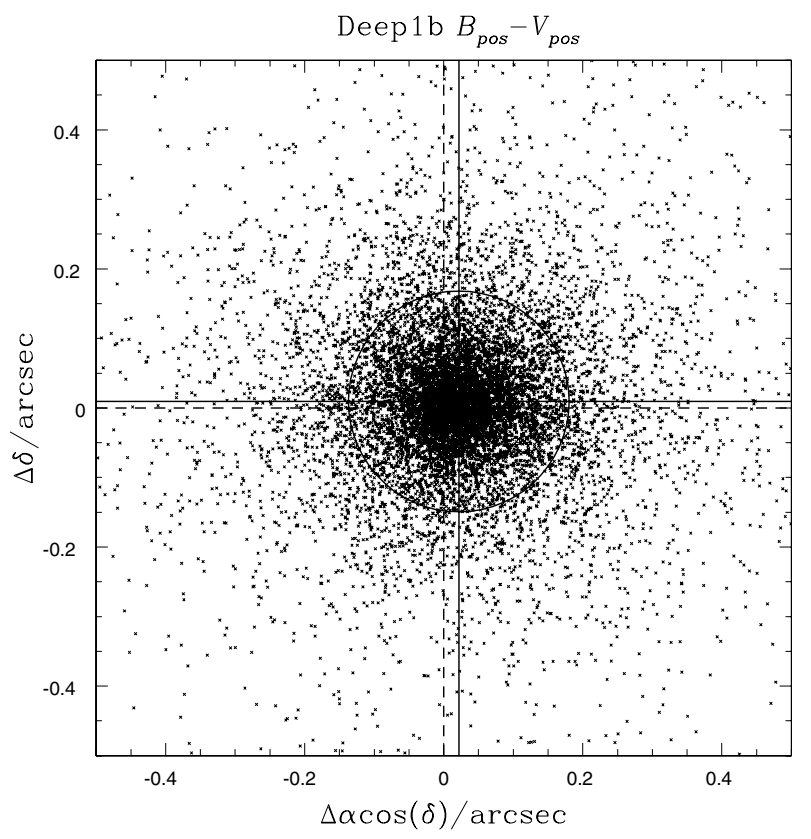

Fig. 6. Distribution of the positional differences of objects in the Deep1b $B$ - and $V$-band images. The mean and standard deviations here are: $\langle\Delta \alpha \cos (\delta)\rangle=0 \prime^{\prime} 02 \pm 00^{\prime} 16$ and $\langle\Delta \delta\rangle=0 \prime^{\prime} 01 \pm 00^{\prime} 15$. The solid lines mark the centre and the circle represents the one sigma interval (radius: $0.15)$.

The external astrometric accuracy is checked with the help of the UCAC-2 catalogue (Zacharias et al. 2004). No offsets larger than the accuracy of the USNO-A2.0 catalogue of 0.3 are found. 


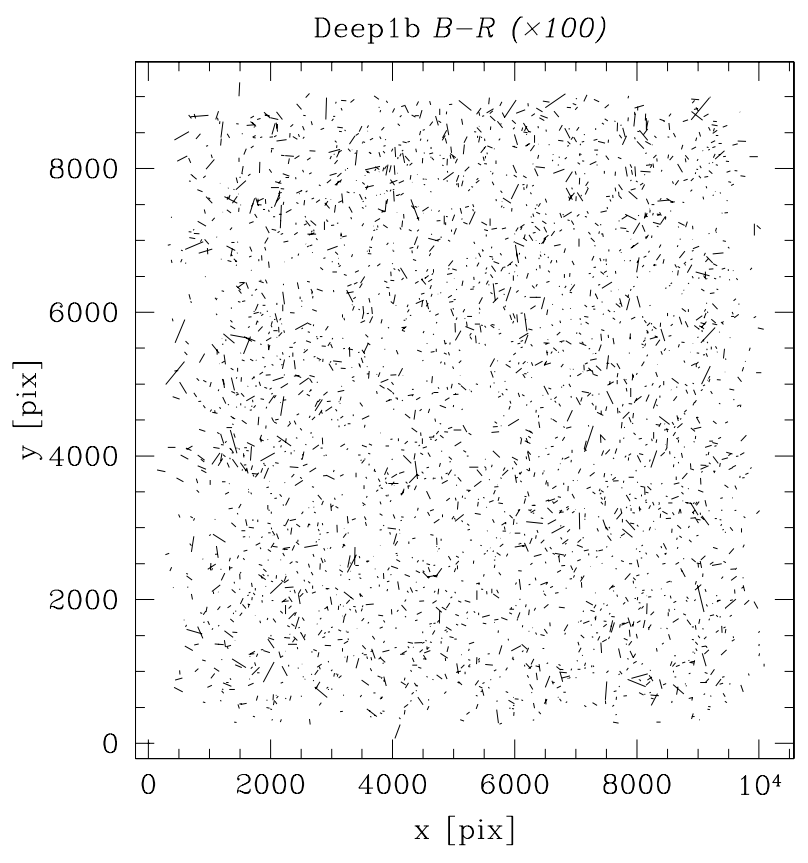

Fig. 7. Dependence of the positional differences on chip position. The distribution seems to be random and no chip boundaries etc. are visible. The differences have been multiplied by a factor 100 and the longest sticks correspond to $\sim 1{ }^{\prime \prime} 5$.

\subsection{Websites}

The released images are accompanied by websites which give quick access to a lot of information about the data reduction. The pages are organised as follows. At the homepage (http://marvin.astro.uni-bonn.de/DPS/) links lead to pages for every filter with further links to all observing runs in this band. These run webpages consist of pages for the calibration frames (BIAS, DARK, SKYFLAT) and for the scientific frames. For the calibration images, thumbnails for visual inspection of the master calibration frame and lists for all single frames used for the creation of the master image are presented. For the scientific frames, basic statistics are listed and a thumbnail of every single frame can be inspected together with a plot of the measured PSF. Further links lead to the photometric solutions of the actual night with plots used for the decision between the different photometric solutions (see Fig. 1).

For every set, websites listing all coadded images in a particular band and their basic properties are also linked from the homepage. Plots showing the distribution of single exposure quantities like seeing, relative photometric zeropoint, etc. are featured as well as colour-colour-plots for all possible combinations. The website for a coadded image contains crosslinks to the run websites of the single frames included and a number of checkplots created after coaddition. In particular, there are checkplots showing the number-counts (see Fig. 4) and angular correlation functions of galaxies in different magnitude bins.

\section{Comparison to the EIS DPS optical data release}

\subsection{Released data}

On November 10, 2004 the EIS team released 40 reduced and stacked optical images of the DPS to the scientific community ${ }^{5}$.

\footnotetext{
5 Available at: http://www.eso.org/science/eis/surveys/ release_60000024_DPS.html
}

Their reduction includes data accumulated until September 28, 2002. Compared to our data release, there are no images for the mispointings, for Deep1a in the $V$-band, for Deep1c in the $I \_E I S$-band, for Deep2c in the $B$ - and $R$-bands, for Deep3a in the $I$-band, and for Deep3d in the $B$ - and $R$-bands. Furthermore, we used more data for the fields Deep1b $(U)$, Deep2b $(I)$, Deep2c (all bands), and Deep3b $(U)$, reaching greater depth. The EIS release includes an image of the field Deep2c in the $U \_38$-band which is not included in our release.

\subsection{Photometric comparison}

After some comparisons between objects' magnitudes in our reduction and in the EIS data release it became clear that the absolute photometric calibration of the EIS images is somewhat different from our calibration. Absolute offsets of some tenths of a magnitude are present for most images. These offsets are also present in colour-colour diagrams when the EIS data are compared to theoretical isochrones by Girardi et al. (2002). Intense cooperation with the EIS team led to the discovery of a mistake in their final application of the absolute photometric calibration to the images (Miralles, private communication) so that no further comparison is meaningful here.

Nevertheless, if both pipelines performed a correct relative photometry between the eight WFI chips there should be only a constant offset between the magnitudes of objects measured in an EIS image and the same objects measured in one of our images. Within photometric errors this is indeed the case for all images from the Deep1 and Deep3 regions. For images from the Deep2 region, the scatter of the magnitude differences is much larger. A careful cooperative analysis with EIS revealed another mistake in the EIS reduction (Miralles, private communication). The chips were not brought to a common zeropoint during the reduction of the Deep2 fields in contrast to Deep1 and Deep3.

\subsection{Astrometric comparison}

The EIS team used the GSC2.2 catalogue for the absolute astrometric calibration which is known to be offset with respect to the USNO-A2.0 catalogue we used. We do not find any offsets between our images and the EIS images of larger than $\sim 0$.' 5 . The actual offset depends strongly on the sky position with the best match in the Deep1 fields and the worst in the Deep3 fields. The scatter is comparable to the scatter between the different colour catalogues from our images (see Sect. 4.3 and Table 4).

Table B. 1 summarises the comparisons between our data release and the EIS data.

\section{Summary}

We release 63 fully reduced and stacked images of the ESO Deep Public Survey to the scientific community. These images, reduced with our THELI pipeline, are shown to have good internal and external astrometric and photometric calibrations. A comparison to the EIS-DPS release shows significant offsets in the absolute photometric calibration for most images and large scatter in the magnitude differences of objects for a subset. Both effects are shown to originate from the EIS data and this is acknowledged by the EIS team.

The optical data from the DPS are currently used by our group to do searches for Lyman-break galaxies. Hildebrandt et al. (2005) have selected large samples of $U$ - and $B$-dropouts 
in deep WFI images of the CDFS. The methods presented there will be applied to the whole DPS dataset.

Acknowledgements. This work was supported by the German Ministry for Education and Science (BMBF) through the DLR under the project 50 OR 0106 , by the BMBF through DESY under the project 05AE2PDA/8, and by the Deutsche Forschungsgemeinschaft (DFG) under the projects SCHN342/3-1 and ER327/2-1.

We would like to thank Dr. L. Girardi very much for creating additional isochrones for the new WFI filters.

Many thanks also to the EIS team for fruitful discussions and comparisons of the two different reductions.

\section{References}

Arnouts, S., Vandame, B., Benoist, C., et al. 2001, A\&A, 379, 740 Bertin, E. 2003, SWarp user's guide
Bertin, E., \& Arnouts, S. 1996, A\&AS, 117, 393

Bolzonella, M., Miralles, J.-M., \& Pelló, R. 2000, A\&A, 363, 476 Bruzual , A. G., \& Charlot, S. 1993, ApJ, 405, 538

Erben, T., Schirmer, M., Dietrich, J. P., et al. 2005, Astron. Nachr., 326, 432

Girardi, L., Bertelli, G., Bressan, A., et al. 2002, A\&A, 391, 195

Greisen, E. W., \& Calabretta, M. R. 2002, A\&A, 395, 1061

Hildebrandt, H., Bomans, D. J., Erben, T., et al. 2005, A\&A, 441, 905

Koch, A., Odenkirchen, M., Grebel, E. K., \& Caldwell, J. A. R. 2004, Astron. Nachr., 325, 299

Landolt, A. U. 1992, AJ, 104, 340

Le Fèvre, O., Vettolani, G., Paltani, S., et al. 2004, A\&A, 428, 1043

Monet, D. B. A., Canzian, B., Dahn, C., et al. 1998, VizieR Online Data Catalog, 1252,0

Prandoni, I., Gregorini, L., Parma, P., et al. 2000, A\&AS, 146, 31

Radovich, M. 2002, ASTROMETRIX

Stetson, P. B. 2000, PASP, 112, 925

Wolf, C., Meisenheimer, K., Kleinheinrich, M., et al. 2004, A\&A, 421, 913

Zacharias, N., Urban, S. E., Zacharias, M. I., et al. 2004, AJ, 127, 3043 
H. Hildebrandt et al.: GaBoDS. V. ESO Deep Public Survey, Online Material p 1

\section{Online Material}


Table 4. Released optical images from the DPS reduced with the THELI pipeline. The first three characters of the coaddition ID in column three represent the field (D1A for field Deep1a etc.) and the fourth character is an identifier to distinguish the different coadditions of one field. The Vega limiting magnitudes in column six correspond to $5 \sigma$ sky level measured in a circular aperture of 2 " diameter. The grades in column seven are estimated from visual inspection of the coadded images. Grade " $\mathrm{A}$ " is assigned to an image with no special features and an appearance typical for the particular band. Grade "B" is assigned to an image with cosmetic defects. Nevertheless also the grade "B" images are fully usable for most scientific purposes. Astrometric offsets in columns eight and nine are given with respect to the $R$-band image (coadd ID D??A and D2CB for the field Deep2c, respectively) of that field.

\begin{tabular}{|c|c|c|c|c|c|c|c|c|}
\hline Field & Filter & coadd ID & $\begin{array}{r}\text { exp. time } \\
{[\mathrm{s}]}\end{array}$ & $\begin{array}{c}F W H M \\
{\left[{ }^{\prime \prime}\right]}\end{array}$ & $\begin{array}{r}\text { mag lim. } \\
\text { [mag] }\end{array}$ & Grade & $\begin{array}{r}\Delta \alpha \cos (\delta) \\
{\left[{ }^{\prime \prime}\right]}\end{array}$ & $\begin{array}{l}\Delta \delta \\
{\left[{ }^{\prime \prime}\right]}\end{array}$ \\
\hline Deep1a & $U \_35060$ & D1AA & 53095 & 1.34 & 25.45 & $\bar{A}$ & $-0.01 \pm 0.16$ & $-0.01 \pm 0.16$ \\
\hline Deep1a & $U \_35060$ & D1AC & 71093 & 1.44 & 25.57 & A & $-0.01 \pm 0.15$ & $-0.01 \pm 0.15$ \\
\hline Deep1a & $U \_38$ & D1AA & 17398 & 1.28 & 24.46 & A & $-0.00 \pm 0.14$ & $-0.01 \pm 0.14$ \\
\hline Deep1a & $B$ & D1AA & 11696 & 1.34 & 26.38 & A & $-0.00 \pm 0.14$ & $-0.01 \pm 0.14$ \\
\hline Deep1a & V & D1AA & 8684 & 0.98 & 25.76 & A & $0.01 \pm 0.12$ & $-0.02 \pm 0.12$ \\
\hline Deep1a & $R$ & D1AA & 9597 & 0.85 & 25.50 & A & - & - \\
\hline Deepla & I & D1AA & 31493 & 0.84 & 24.33 & A & $0.00 \pm 0.12$ & $-0.00 \pm 0.11$ \\
\hline Deep1a & $I$ & D1AG & 25194 & 0.84 & 24.29 & A & $0.00 \pm 0.12$ & $-0.00 \pm 0.11$ \\
\hline Deep1b & $\overline{U \_35060}$ & $\overline{\mathrm{D} 1 \mathrm{BA}}$ & 46793 & 1.06 & 25.27 & $\overline{\mathrm{A}}$ & $-0.03 \pm 0.15$ & $-0.01 \pm 0.15$ \\
\hline Deep1b & $B$ & D1BA & 9597 & 1.33 & 26.09 & A & $-0.02 \pm 0.15$ & $-0.01 \pm 0.14$ \\
\hline Deep1b & $B$ & D1BS & 4199 & 1.09 & 25.61 & A & $-0.02 \pm 0.13$ & $-0.00 \pm 0.13$ \\
\hline Deep1b & $V$ & D1BA & 9897 & 1.32 & 25.60 & A & $-0.00 \pm 0.14$ & $0.00 \pm 0.13$ \\
\hline Deep1b & $V$ & D1BS & 4499 & 1.20 & 25.24 & A & $0.00 \pm 0.13$ & $-0.00 \pm 0.12$ \\
\hline Deep1b & $R$ & D1BA & 19794 & 1.28 & 25.70 & B & - & - \\
\hline Deep1b & $R$ & D1BS & 8398 & 1.10 & 25.28 & A & $0.00 \pm 0.10$ & $0.00 \pm 0.09$ \\
\hline Deep1b & $I$ & D1BA & 25493 & 0.97 & 24.38 & A & $-0.01 \pm 0.13$ & $-0.00 \pm 0.12$ \\
\hline Deep1c & $V$ & D1CA & 7498 & 1.19 & 25.47 & $\bar{A}$ & $-0.03 \pm 0.16$ & $-0.00 \pm 0.15$ \\
\hline Deep1c & $V$ & D1CS & 4199 & 1.11 & 25.25 & A & $-0.03 \pm 0.15$ & $-0.00 \pm 0.14$ \\
\hline Deep1c & $R$ & D1CA & 11696 & 0.97 & 25.28 & A & - & - \\
\hline Deep1c & $I \_E I S$ & D1CA & 4445 & 1.25 & 23.34 & A & $-0.04 \pm 0.15$ & $-0.02 \pm 0.14$ \\
\hline Deep1e & $\bar{U} U 38$ & D1EA & 9899 & 1.78 & 24.34 & $\bar{A}$ & $-0.01 \pm 0.09$ & $-0.03 \pm 0.21$ \\
\hline Deep1e & $R$ & D1EA & 8998 & 0.90 & 25.37 & A & - & - \\
\hline Deep1e & $I$ & D1EA & 11398 & 1.26 & 23.93 & A & $-0.01 \pm 0.07$ & $-0.02 \pm 0.15$ \\
\hline Deep1f & $I$ & D1FA & 14997 & 1.16 & 23.92 & $\bar{A}$ & - & - \\
\hline Deep2a & $R$ & D2AA & 5998 & 0.83 & 25.11 & $\mathrm{~A}$ & - & - \\
\hline Deep2b & $U \_35060$ & D2BA & 53095 & 1.15 & 25.23 & A & $-0.02 \pm 0.14$ & $-0.01 \pm 0.14$ \\
\hline Deep2b & $U \_35060$ & $\mathrm{D} 2 \mathrm{BC}$ & 64794 & 1.27 & 25.30 & A & $-0.02 \pm 0.14$ & $-0.01 \pm 0.14$ \\
\hline Deep2b & $B$ & D2BA & 11396 & 0.98 & 26.23 & A & $0.00 \pm 0.13$ & $0.00 \pm 0.13$ \\
\hline Deep2b & $B$ & D2BS & 9597 & 0.91 & 26.13 & A & $0.00 \pm 0.13$ & $0.00 \pm 0.13$ \\
\hline Deep2b & $V$ & D2BA & 9297 & 0.88 & 25.59 & A & $-0.00 \pm 0.12$ & $0.01 \pm 0.12$ \\
\hline Deep2b & $R$ & D2BA & 10497 & 1.33 & 25.42 & A & - & - \\
\hline Deep2b & $R$ & D2BS & 4799 & 1.09 & 25.01 & A & $0.00 \pm 0.08$ & $0.00 \pm 0.08$ \\
\hline Deep2b & $I$ & D2BA & 19196 & 0.75 & 24.25 & A & $0.00 \pm 0.12$ & $0.01 \pm 0.11$ \\
\hline Deep2c & $U_{-} 35060$ & $\mathrm{D} 2 \mathrm{CB}$ & 78891 & 1.01 & 25.49 & A & $-0.02 \pm 0.13$ & $0.03 \pm 0.14$ \\
\hline Deep2c & $B$ & D2CA & 69431 & 0.98 & 27.28 & A & $0.01 \pm 0.11$ & $-0.00 \pm 0.11$ \\
\hline Deep2c & $V$ & D2CB & 104603 & 0.92 & 26.77 & A & $0.01 \pm 0.10$ & $0.00 \pm 0.10$ \\
\hline Deep2c & $R$ & D2CB & 87654 & 0.79 & 26.54 & A & - & - \\
\hline Deep2c & $I$ & D2CA & 34575 & 0.93 & 24.49 & A & $0.00 \pm 0.11$ & $0.00 \pm 0.11$ \\
\hline Deep2d & $R$ & D2DA & 2999 & 1.06 & 24.81 & $\mathrm{~A}$ & - & - \\
\hline Deep3a & $\bar{U} U 35060$ & D3AA & 26997 & 1.09 & 24.77 & $\overline{\mathrm{A}}$ & $-0.01 \pm 0.13$ & $-0.01 \pm 0.13$ \\
\hline Deep3a & $U \_35060$ & D3AC & 35996 & 1.10 & 24.91 & A & $-0.01 \pm 0.13$ & $-0.00 \pm 0.13$ \\
\hline Deep3a & $U \_38$ & D3AA & 26997 & 1.27 & 24.59 & A & $-0.01 \pm 0.12$ & $-0.00 \pm 0.12$ \\
\hline Deep3a & $B$ & D3AA & 11096 & 0.92 & 26.08 & A & $-0.00 \pm 0.12$ & $-0.00 \pm 0.11$ \\
\hline Deep3a & $V$ & D3AA & 8998 & 1.02 & 25.44 & A & $-0.00 \pm 0.11$ & $-0.00 \pm 0.11$ \\
\hline Deep3a & $R$ & D3AA & 8997 & 0.81 & 25.28 & A & - & - \\
\hline Deep3a & $R$ & D3AS & 7798 & 0.79 & 25.23 & A & $-0.00 \pm 0.05$ & $0.00 \pm 0.05$ \\
\hline Deep3a & $I$ & D3AA & 23396 & 0.95 & 24.22 & B & $0.01 \pm 0.11$ & $0.00 \pm 0.11$ \\
\hline Deep3a & $I$ & D3AG & 13198 & 0.91 & 23.94 & A & $0.00 \pm 0.11$ & $0.00 \pm 0.11$ \\
\hline
\end{tabular}


H. Hildebrandt et al.: GaBoDS. V. ESO Deep Public Survey, Online Material p 3

Table 4. continued.

\begin{tabular}{|c|c|c|c|c|c|c|c|c|}
\hline Field & Filter & coadd ID & $\begin{array}{r}\text { exp. time } \\
{[\mathrm{s}]}\end{array}$ & $\begin{array}{c}F W H M \\
{\left[{ }^{\prime \prime}\right]}\end{array}$ & $\begin{array}{r}\text { mag lim. } \\
{[\mathrm{mag}]}\end{array}$ & Grade & $\begin{array}{r}\Delta \alpha \cos (\delta) \\
{\left[{ }^{\prime \prime}\right]}\end{array}$ & $\begin{array}{r}\Delta \delta \\
{\left[{ }^{\prime \prime}\right]}\end{array}$ \\
\hline Deep3b & $\bar{U} U 35060$ & $\overline{\mathrm{D} 3 \mathrm{BA}}$ & 53995 & 1.01 & 25.13 & $\bar{A}$ & $0.01 \pm 0.13$ & $-0.00 \pm 0.13$ \\
\hline Deep3b & $B$ & D3BA & 9897 & 0.95 & 26.06 & A & $0.00 \pm 0.12$ & $-0.00 \pm 0.12$ \\
\hline Deep3b & $V$ & D3BA & 8998 & 0.88 & 25.45 & A & $0.00 \pm 0.11$ & $0.00 \pm 0.11$ \\
\hline Deep3b & $R$ & D3BA & 9297 & 0.79 & 25.30 & A & - & - \\
\hline Deep3b & $I$ & D3BA & 26993 & 0.82 & 24.27 & A & $0.00 \pm 0.11$ & $0.01 \pm 0.10$ \\
\hline Deep3c & $U \_35060$ & $\overline{\mathrm{D} 3 \mathrm{CA}}$ & 46845 & 0.97 & 25.22 & $\overline{\mathrm{A}}$ & $0.01 \pm 0.13$ & $-0.00 \pm 0.13$ \\
\hline Deep3c & $B$ & D3CA & 13496 & 0.93 & 26.24 & A & $0.01 \pm 0.12$ & $0.00 \pm 0.12$ \\
\hline Deep3c & $B$ & D3CS & 11996 & 0.90 & 26.17 & A & $0.01 \pm 0.12$ & $-0.00 \pm 0.12$ \\
\hline Deep3c & V & D3CA & 5998 & 0.79 & 25.30 & A & $0.01 \pm 0.11$ & $-0.00 \pm 0.10$ \\
\hline Deep3c & $R$ & D3CA & 8998 & 0.81 & 25.26 & A & - & - \\
\hline Deep3c & $I$ & D3CA & 25193 & 1.02 & 24.04 & B & $0.01 \pm 0.11$ & $0.00 \pm 0.11$ \\
\hline Deep3d & $\bar{B}$ & $\overline{\mathrm{D} 3 \mathrm{DA}}$ & 11097 & 0.88 & 26.22 & $\bar{A}$ & $0.01 \pm 0.12$ & $-0.01 \pm 0.12$ \\
\hline Deep3d & $V$ & D3DA & 8998 & 0.90 & 25.37 & A & $0.01 \pm 0.11$ & $-0.00 \pm 0.10$ \\
\hline Deep3d & $R$ & D3DA & 8998 & 0.73 & 24.76 & A & - & - \\
\hline Deep3d & I_EIS & D3DA & 22293 & 0.76 & 24.51 & A & $0.01 \pm 0.10$ & $-0.00 \pm 0.10$ \\
\hline
\end{tabular}




\section{Appendix A: Photometric calibrations}

In Tables A.1 to A.7 the photometric solutions for all calibrated nights are shown. The solution chosen for a particular coadded image can be found in the FITS image header.

\section{Appendix B: Comparison to the EIS data}

In Table B.1 the released data from the EIS team are compared to our data.

\section{Appendix C: FITS header}

In the following we show an excerpt of one of our image headers to describe the special keywords inserted by the reduction pipeline $^{6}$. EXPTIME is the sum of the exposure times of all images that entered the coaddition. GAIN is the instrumental gain (2.0) multiplied by EXPTIME. MAGZP is the Vega magnitude zeropoint which is to be used for converting counts into magnitudes by: mag = MAGZP $-2.5 \cdot \log$ (counts). The COND keywords contain the filter conditions that were applied to the image catalogues before coaddition. Within these conditions AUTO_ZP represents a filtering on the single images' relative zeropoints, BACKGR a filtering on the single images' background flux, and SEEING a filtering on the single images' measured seeing. The NIGHT keywords summarise the absolute photometric calibration containing pairs of GaBoDS IDs and chosen solutions, e.g. 9032 means that in the night number 903 (2001-06-21) the two-parameter fit was chosen.

In Table C. 1 the important FITS header keywords of our released images are summarised.

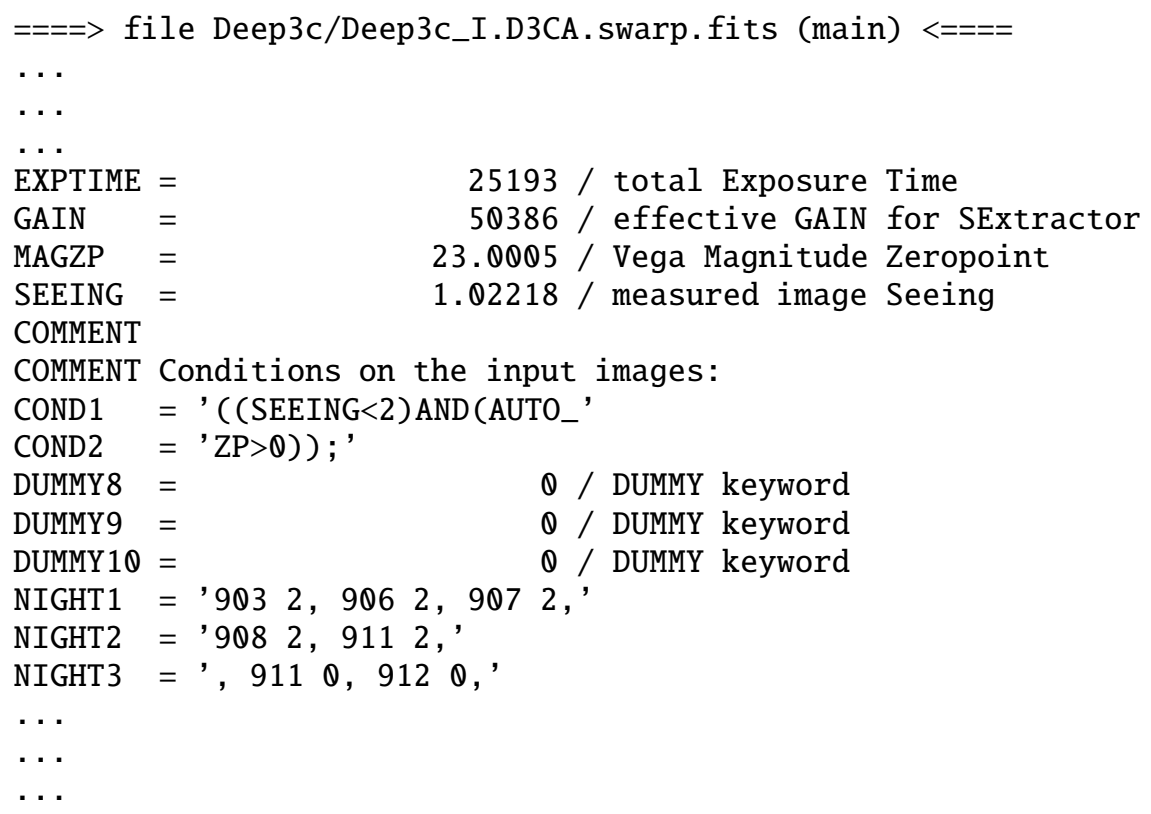

\footnotetext{
6 The image headers of the files released via the ESO archive at http://archive.eso.org/archive/eso_data_products.html are slightly different and contain a lot of additional keywords in order to comply with archive standards.
} 
H. Hildebrandt et al.: GaBoDS. V. ESO Deep Public Survey, Online Material p 5

Table A.1. Photometric solutions in the $U \_35060$-band. The colour term corresponds to the Johnson-Cousins colour $U-B$. The default value for the extinction coefficient in the one- and two-parameter fits is EXT1 $=$ EXT2 $=-0.48$ and the default value for the colour term in the one-parameter fit is $\mathrm{CT} 1=0.05$.

\begin{tabular}{|c|c|c|c|c|c|c|c|}
\hline night & GaBoDS ID & ZP3 & EXT3 & CT3 & $\mathrm{ZP2}$ & CT2 & ZP1 \\
\hline $2000-10-26$ & 665 & 20.48 & -0.00 & -10.04 & 21.15 & -10.21 & 22.06 \\
\hline $2000-10-27$ & 666 & 22.11 & -0.47 & -0.11 & 22.12 & -0.11 & 22.12 \\
\hline $2000-11-27$ & 697 & 23.81 & -1.93 & 0.02 & 22.13 & 0.02 & 22.11 \\
\hline $2000-11-28$ & 698 & 27.06 & -4.64 & 0.05 & 22.12 & 0.05 & 22.12 \\
\hline $2001-02-21$ & 783 & 21.21 & 0.00 & 0.02 & 21.79 & 0.02 & 21.77 \\
\hline $2001-02-22$ & 784 & 21.35 & 0.00 & 0.09 & 21.95 & 0.10 & 21.97 \\
\hline $2001-02-23$ & 785 & 21.78 & -0.26 & 0.10 & 22.07 & 0.10 & 22.09 \\
\hline 2001-02-26 & 788 & 21.85 & -0.37 & 0.08 & 21.99 & 0.08 & 22.00 \\
\hline $2001-03-24$ & 814 & 22.19 & -0.62 & 0.07 & 22.02 & 0.07 & 22.03 \\
\hline $2001-03-25$ & 815 & 22.55 & -0.94 & 0.09 & 21.99 & 0.09 & 22.00 \\
\hline 2001-04-20 & 841 & 23.09 & -1.38 & 0.10 & 21.78 & 0.10 & 21.80 \\
\hline 2001-04-21 & 842 & 21.88 & -0.36 & 0.07 & 22.04 & 0.06 & 22.04 \\
\hline 2001-07-20 & 932 & 25.73 & -3.23 & 0.23 & 22.04 & 0.22 & 22.17 \\
\hline $2001-07-21$ & 933 & 23.36 & -1.51 & 0.37 & 21.92 & 0.44 & 22.11 \\
\hline $2001-07-22$ & 934 & 22.82 & -0.87 & 0.14 & 22.33 & 0.13 & 22.38 \\
\hline $2001-07-23$ & 935 & 21.58 & -0.00 & 0.11 & 22.21 & 0.11 & 22.26 \\
\hline $2001-07-24$ & 936 & 22.24 & -0.40 & 0.19 & 22.34 & 0.19 & 22.41 \\
\hline $2001-07-25$ & 937 & - & - & - & 22.05 & 0.41 & 22.31 \\
\hline $2001-07-26$ & 938 & - & - & - & 21.92 & 0.52 & 22.21 \\
\hline 2001-08-19 & 962 & 22.46 & -0.54 & 0.08 & 22.37 & 0.08 & 22.38 \\
\hline 2001-08-20 & 963 & 22.40 & -0.51 & 0.09 & 22.37 & 0.09 & 22.39 \\
\hline $2001-08-21$ & 964 & 22.90 & -0.91 & 0.04 & 22.37 & 0.05 & 22.37 \\
\hline 2001-08-22 & 965 & 26.52 & -3.95 & 0.02 & 22.35 & 0.05 & 22.35 \\
\hline $2001-08-23$ & 966 & 22.35 & -0.48 & 0.06 & 22.35 & 0.06 & 22.35 \\
\hline 2001-11-13 & 1048 & 21.73 & 0.00 & 0.01 & 22.29 & 0.01 & 22.27 \\
\hline 2001-11-14 & 1049 & 22.97 & -1.06 & 0.08 & 22.29 & 0.09 & 22.31 \\
\hline $2001-11-15$ & 1050 & 22.78 & -0.90 & 0.05 & 22.28 & 0.05 & 22.28 \\
\hline 2002-03-09 & 1164 & 22.10 & -0.47 & 0.04 & 22.12 & 0.04 & 22.12 \\
\hline 2002-03-10 & 1165 & 22.01 & -0.45 & 0.16 & 22.04 & 0.16 & 22.10 \\
\hline $2002-03-11$ & 1166 & 21.95 & -0.28 & 0.11 & 22.20 & 0.12 & 22.23 \\
\hline 2002-03-12 & 1167 & 22.12 & -0.37 & 0.05 & 22.26 & 0.06 & 22.26 \\
\hline $2002-03-13$ & 1168 & 21.73 & 0.00 & 0.03 & 22.28 & 0.03 & 22.27 \\
\hline 2002-06-07 & 1254 & 21.49 & 0.00 & 0.57 & 22.07 & 0.56 & 22.33 \\
\hline 2002-06-09 & 1256 & 22.88 & -1.00 & -0.11 & 22.24 & -0.08 & 22.23 \\
\hline 2002-06-10 & 1257 & 22.18 & -0.39 & 0.08 & 22.28 & 0.08 & 22.29 \\
\hline $2002-06-12$ & 1259 & - & - & - & 21.82 & 2.50 & 22.06 \\
\hline 2002-08-11 & 1319 & 22.02 & -0.35 & -0.69 & 22.18 & -0.71 & 21.99 \\
\hline $2002-09-28$ & 1367 & - & - & - & 21.86 & 0.70 & 22.20 \\
\hline $2002-12-11$ & 1441 & 22.28 & -0.54 & 0.12 & 22.20 & 0.12 & 22.24 \\
\hline 2004-05-12 & 1959 & 21.58 & 0.00 & -0.14 & 22.15 & -0.14 & 22.12 \\
\hline 2004-10-31 & 2131 & 23.72 & -1.67 & 0.02 & 22.35 & 0.03 & 22.35 \\
\hline 2004-11-02 & 2133 & - & - & - & - & - & 22.32 \\
\hline $2005-10-30$ & 2495 & 21.81 & -0.09 & 0.07 & 22.40 & 0.00 & 22.36 \\
\hline
\end{tabular}

Table A.2. Photometric solutions in the $U \_38$-band. The colour term corresponds to the Johnson-Cousins colour $U-B$. The default value for the extinction coefficient in the one- and two-parameter fits is EXT1 $=$ EXT2 $=-0.73$ and the default value for the colour term in the one-parameter fit is $\mathrm{CT} 1=-0.01$.

\begin{tabular}{cr|rrr|rr|r}
\hline \hline night & GaBoDS ID & ZP3 & EXT3 & CT3 & ZP2 & CT2 & ZP1 \\
\hline $2000-03-30$ & 455 & 21.24 & 0.00 & 0.03 & 22.09 & 0.03 & 22.11 \\
$2000-03-31$ & 456 & 21.21 & 0.00 & 0.05 & 22.07 & 0.05 & 22.10 \\
$2000-04-01$ & 457 & 25.19 & -3.37 & 0.05 & 22.09 & 0.05 & 22.12 \\
$2000-04-05$ & 461 & 21.17 & 0.00 & 0.04 & 22.03 & 0.04 & 22.05 \\
$2000-07-29$ & 576 & - & - & - & 23.76 & 16.57 & 22.15 \\
$2000-08-01$ & 579 & - & - & - & 23.09 & 9.37 & 22.18 \\
$2000-08-26$ & 604 & 21.34 & -0.06 & 0.09 & 22.17 & 0.08 & 22.24 \\
$2000-08-27$ & 605 & 20.97 & 0.00 & 0.55 & 21.84 & 0.55 & 22.17 \\
\hline
\end{tabular}


H. Hildebrandt et al.: GaBoDS. V. ESO Deep Public Survey, Online Material p 6

Table A.3. Photometric solutions in the $B$-band. The colour term corresponds to the Johnson-Cousins colour $B-V$. The default value for the extinction coefficient in the one- and two-parameter fits is EXT1 $=$ EXT2 $=-0.22$ and the default value for the colour term in the one-parameter fit is $\mathrm{CT} 1=0.25$.

\begin{tabular}{cr|rrr|rr|r}
\hline \hline night & GaBoDS ID & ZP3 & EXT3 & CT3 & ZP2 & CT2 & ZP1 \\
\hline $1999-12-02$ & 336 & - & - & - & 24.74 & 0.27 & 24.76 \\
$2000-03-29$ & 454 & 25.61 & -0.99 & 0.27 & 24.59 & 0.26 & 24.60 \\
$2000-03-30$ & 455 & 24.72 & -0.29 & 0.23 & 24.63 & 0.23 & 24.61 \\
$2000-03-31$ & 456 & 24.83 & -0.38 & 0.23 & 24.63 & 0.23 & 24.62 \\
$2000-04-01$ & 457 & 24.40 & 0.00 & 0.21 & 24.66 & 0.21 & 24.63 \\
$2000-04-06$ & 462 & 24.43 & -0.00 & 0.20 & 24.69 & 0.20 & 24.65 \\
$2000-07-02$ & 549 & 26.29 & -1.53 & 0.17 & 24.77 & 0.18 & 24.69 \\
$2000-11-27$ & 697 & 24.42 & 0.00 & 0.22 & 24.70 & 0.22 & 24.68 \\
$2000-11-28$ & 698 & 24.71 & -0.23 & 0.22 & 24.69 & 0.22 & 24.67 \\
$2001-02-01$ & 763 & 24.66 & -0.23 & 0.23 & 24.65 & 0.23 & 24.63 \\
$2001-02-02$ & 764 & 24.45 & -0.16 & 0.23 & 24.53 & 0.23 & 24.51 \\
$2001-02-25$ & 787 & 24.66 & -0.27 & 0.23 & 24.58 & 0.23 & 24.57 \\
$2001-02-26$ & 788 & 24.51 & -0.14 & 0.22 & 24.61 & 0.22 & 24.59 \\
$2001-11-13$ & 1048 & 24.49 & 0.00 & 0.21 & 24.75 & 0.21 & 24.71 \\
$2001-11-14$ & 1049 & 24.48 & 0.00 & 0.22 & 24.74 & 0.22 & 24.72 \\
$2001-11-15$ & 1050 & 24.69 & -0.18 & 0.23 & 24.74 & 0.23 & 24.72 \\
$2001-12-12$ & 1077 & 24.80 & -0.25 & 0.22 & 24.76 & 0.22 & 24.74 \\
$2002-02-01$ & 1128 & 22.54 & -0.00 & 1.29 & 22.80 & 1.29 & 23.80 \\
$2002-02-02$ & 1129 & 24.72 & -0.25 & 0.26 & 24.67 & 0.27 & 24.69 \\
$2002-02-03$ & 1130 & 24.70 & -0.21 & 0.22 & 24.71 & 0.22 & 24.69 \\
$2002-02-04$ & 1131 & 24.63 & -0.18 & 0.21 & 24.68 & 0.21 & 24.65 \\
$2002-02-05$ & 1132 & 25.01 & -0.50 & 0.20 & 24.66 & 0.21 & 24.63 \\
$2002-06-07$ & 1254 & 24.85 & -0.30 & 0.19 & 24.75 & 0.19 & 24.69 \\
$2002-10-12$ & 1381 & 24.56 & -0.14 & 0.25 & 24.66 & 0.25 & 24.66 \\
$2004-01-31$ & 1857 & 24.41 & 0.00 & 0.22 & 24.67 & 0.22 & 24.64 \\
$2004-02-01$ & 1858 & 24.39 & 0.00 & 0.23 & 24.65 & 0.23 & 24.63 \\
$2004-03-19$ & 1905 & - & - & - & 24.61 & 0.29 & 24.63 \\
\hline
\end{tabular}


H. Hildebrandt et al.: GaBoDS. V. ESO Deep Public Survey, Online Material p 7

Table A.4. Photometric solutions in the $V$-band. The colour term corresponds to the Johnson-Cousins colour $V-R$. The default value for the extinction coefficient in the one- and two-parameter fits is EXT1 $=$ EXT2 $=-0.11$ and the default value for the colour term in the one-parameter fit is $\mathrm{CT} 1=-0.13$.

\begin{tabular}{|c|c|c|c|c|c|c|c|}
\hline Night & GaBoDS ID & ZP3 & EXT3 & CT3 & $\mathrm{ZP} 2$ & CT2 & ZP1 \\
\hline 1999-11-08 & 312 & - & - & - & 24.37 & -0.16 & 24.35 \\
\hline 1999-12-02 & 336 & 24.49 & -0.33 & -0.12 & 24.27 & -0.12 & 24.27 \\
\hline $1999-12-03$ & 337 & 24.34 & -0.17 & -0.18 & 24.27 & -0.18 & 24.24 \\
\hline 1999-1 & 338 & 24.23 & -0.07 & -0.17 & 24.27 & -0.17 & 24.25 \\
\hline 2000-03-29 & 454 & 24.24 & -0 . & -0.17 & 24.22 & -0.17 & 24.20 \\
\hline $2000-0$ & 455 & 4.40 & -0.30 & -0.12 & 24.17 & -0.11 & 24.18 \\
\hline 2000-03-31 & 456 & 24.51 & -0.42 & -0.12 & 24.13 & -0.11 & 24.14 \\
\hline 2000-04-01 & 457 & 23.89 & 0.00 & -0.07 & 24.02 & -0.07 & 24.05 \\
\hline 2000-04-06 & 462 & 24.09 & 0.00 & -0.13 & 24.22 & -0.14 & 24.22 \\
\hline $2000-07-03$ & 550 & 22.81 & 0.00 & -0.93 & 22.94 & -0.93 & 22.12 \\
\hline $2000-11-28$ & 698 & 24.09 & 0.00 & -0.16 & 24.23 & -0.16 & 24.21 \\
\hline $2000-11-29$ & 699 & 24.30 & -0.17 & -0.16 & 24.22 & -0.16 & 24.20 \\
\hline $2001-02-02$ & 764 & 24.08 & -0.10 & -0.15 & 24.10 & -0.16 & 24.09 \\
\hline $2001-02-20$ & 782 & 24.39 & -0.34 & -0.15 & 24.08 & -0.13 & 24.08 \\
\hline $2001-02-23$ & 785 & 24.07 & 0.00 & -0.16 & 24.21 & -0.16 & 24.19 \\
\hline $2001-02-25$ & 787 & 24.25 & -0.19 & -0.16 & 24.14 & -0.16 & 24.13 \\
\hline 2001 & 788 & 24.15 & -0.09 & -0.15 & 24.17 & -0.15 & 24.16 \\
\hline 2001 & 816 & 4.53 & -0.45 & -0.18 & 23.99 & -0.04 & 24.04 \\
\hline 2001 & 817 & 4.04 & 0.00 & -0.17 & 24.17 & -0.17 & 24.15 \\
\hline 2001 & 907 & 24.12 & 0.00 & -0.15 & 24.26 & -0.16 & 24.24 \\
\hline 2001 & 909 & 24.42 & -0.22 & -0.18 & 24.25 & -0.15 & 24.23 \\
\hline 2001 & 911 & 24.28 & -0.11 & -0.16 & 24.28 & -0.16 & 24.25 \\
\hline 2001-( & 938 & 24.36 & -0.13 & -0.19 & 24.34 & -0.19 & 24.27 \\
\hline 2001-( & 964 & 24.31 & -0.13 & -0.14 & 24.28 & -0.14 & 24.27 \\
\hline 2001- & 965 & 24.30 & -0.15 & -0.13 & 24.25 & -0.14 & 24.24 \\
\hline $2001-0$ & 966 & 5.26 & -0.94 & -0.17 & 24.25 & -0.14 & 24.24 \\
\hline 2001-1 & 1047 & 4.07 & -0.00 & -0.23 & 24.20 & -0.23 & 24.15 \\
\hline 2001-1 & 54 & 4.24 & -0.05 & -0.20 & 24.31 & -0.20 & 24.28 \\
\hline 2001-1 & 73 & 24.27 & -0.10 & -0.22 & 24.29 & -0.22 & 24.25 \\
\hline 2001-1 & 78 & 4.35 & -0.16 & & 24.28 & -0.15 & 24.26 \\
\hline $2002-0$ & 36 & .94 & 0.00 & & 24.07 & -0.17 & 24.05 \\
\hline & & .08 & -0.09 & & 24.10 & -0.14 & 24.09 \\
\hline & & & -0.38 & & 24.22 & -0 & 24.21 \\
\hline & & 1.34 & -0.21 & & 24.22 & -0 & 24.22 \\
\hline & & 4.06 & 0.00 & & 24.19 & -0.15 & 24.18 \\
\hline & & 24.30 & -0.18 & & 24.20 & -0.15 & 24.19 \\
\hline 2002- & 381 & 24.21 & -0.13 & -0.16 & 24.19 & -0.16 & 24.17 \\
\hline 2004-( & 183 & 24.18 & -0.12 & -0.15 & 24.17 & -0.15 & 24.15 \\
\hline 2004-0 & 184 & 24.19 & -0.15 & -0.15 & 24.14 & -0.15 & 24.13 \\
\hline 2004- & 7 & 24.15 & -0.11 & -0.15 & 24.15 & -0.15 & 24.14 \\
\hline 2004 & 2102 & 24.06 & 0.00 & -0.11 & 24.19 & -0.11 & 24.20 \\
\hline 2004- 1 & 2107 & - & - & - & 23.92 & -0.26 & 23.86 \\
\hline 2005-( & 2465 & 24.24 & -0.10 & -0.17 & 24.25 & -0.17 & 24.21 \\
\hline $2005-10-30$ & 2495 & 24.36 & -0.20 & -0.20 & 24.23 & -0.18 & 24.20 \\
\hline
\end{tabular}


H. Hildebrandt et al.: GaBoDS. V. ESO Deep Public Survey, Online Material $p 8$

Table A.5. Photometric solutions in the $R$-band. The colour term corresponds to the Johnson-Cousins colour $V-R$. The default value for the extinction coefficient in the one- and two-parameter fits is EXT1 $=$ EXT2 $=-0.07$ and the default value for the colour term in the one-parameter fit is $\mathrm{CT} 1=0.00$.

\begin{tabular}{|c|c|c|c|c|c|c|c|}
\hline Night & GaBoDS ID & ZP3 & EXT3 & CT3 & ZP2 & CT2 & ZP1 \\
\hline 1999-12-04 & 338 & 24.47 & 0.00 & -0.06 & 24.55 & -0.06 & 24.51 \\
\hline 2000-03-29 & 454 & 24.46 & 0.00 & -0.09 & 24.55 & -0.09 & 24.50 \\
\hline 2000-03-30 & 455 & 24.39 & 0.00 & -0.05 & 24.47 & -0.05 & 24.44 \\
\hline 2000-03-31 & 456 & 24.32 & 0.00 & -0.03 & 24.40 & -0.03 & 24.39 \\
\hline 2000-04-05 & 461 & 24.49 & -0.06 & -0.02 & 24.50 & -0.02 & 24.49 \\
\hline 2000-04-06 & 462 & 24.44 & 0.00 & -0.02 & 24.52 & -0.02 & 24.51 \\
\hline $2000-07-27$ & 574 & 24.54 & 0.00 & -0.12 & 24.63 & -0.12 & 24.55 \\
\hline $2000-08-25$ & 603 & 25.04 & -0.38 & -0.15 & 24.59 & -0.09 & 24.49 \\
\hline $2000-08-26$ & 604 & 24.88 & -0.27 & -0.15 & 24.63 & -0.14 & 24.47 \\
\hline $2000-08-27$ & 605 & 25.04 & -0.38 & -0.13 & 24.66 & -0.12 & 24.50 \\
\hline $2000-12-25$ & 725 & 24.46 & 0.00 & -0.01 & 24.54 & -0.01 & 24.53 \\
\hline $2000-12-26$ & 726 & 24.54 & -0.08 & -0.02 & 24.53 & -0.01 & 24.52 \\
\hline $2001-02-20$ & 782 & 24.45 & -0.09 & -0.02 & 24.42 & -0.02 & 24.41 \\
\hline $2001-02-22$ & 784 & 24.36 & 0.00 & -0.04 & 24.44 & -0.04 & 24.42 \\
\hline $2001-02-23$ & 785 & 24.42 & 0.00 & -0.03 & 24.51 & -0.04 & 24.49 \\
\hline 2001-03-26 & 816 & 24.67 & -0.28 & -0.03 & 24.28 & 0.06 & 24.31 \\
\hline $2001-03-27$ & 817 & 24.42 & 0.00 & -0.07 & 24.51 & -0.07 & 24.47 \\
\hline 2001-06-20 & 902 & 24.49 & 0.00 & -0.06 & 24.60 & -0.07 & 24.56 \\
\hline 2001-06-21 & 903 & 24.49 & 0.00 & -0.05 & 24.60 & -0.05 & 24.57 \\
\hline 2001-06-26 & 908 & 24.18 & 0.00 & 0.29 & 24.28 & 0.29 & 24.41 \\
\hline 2001-06-27 & 909 & 24.70 & -0.11 & -0.12 & 24.64 & -0.12 & 24.55 \\
\hline $2001-06-29$ & 911 & 24.65 & -0.08 & -0.12 & 24.64 & -0.12 & 24.50 \\
\hline 2001-06-30 & 912 & 24.39 & 0.00 & -0.02 & 24.48 & -0.02 & 24.47 \\
\hline $2001-08-20$ & 963 & 24.64 & -0.07 & -0.10 & 24.64 & -0.10 & 24.54 \\
\hline 2001-08-21 & 964 & 24.67 & -0.09 & -0.10 & 24.64 & -0.10 & 24.54 \\
\hline 2001-11-12 & 1047 & 24.39 & -0.00 & -0.01 & 24.47 & -0.01 & 24.47 \\
\hline 2001-11-16 & 1051 & 24.58 & -0.06 & -0.11 & 24.59 & -0.11 & 24.52 \\
\hline 2001-11-17 & 1052 & 24.62 & -0.11 & -0.09 & 24.58 & -0.10 & 24.53 \\
\hline 2001-11-19 & 1054 & 24.53 & 0.00 & -0.08 & 24.61 & -0.08 & 24.57 \\
\hline 2001-12-09 & 1074 & 24.50 & 0.00 & -0.09 & 24.58 & -0.09 & 24.54 \\
\hline $2001-12-11$ & 1076 & 24.55 & -0.05 & -0.05 & 24.57 & -0.05 & 24.54 \\
\hline 2003-04-05 & 1556 & - & - & - & 24.47 & -0.05 & 24.43 \\
\hline 2003-04-06 & 1557 & 24.40 & 0.00 & -0.06 & 24.48 & -0.06 & 24.44 \\
\hline 2003-04-21 & 1572 & 24.58 & -0.08 & -0.12 & 24.56 & -0.12 & 24.51 \\
\hline 2004-10-04 & 2104 & 24.26 & 0.00 & -0.06 & 24.44 & -0.06 & 24.40 \\
\hline 2004-10-09 & 2109 & 24.32 & 0.00 & -0.08 & 24.40 & -0.08 & 24.36 \\
\hline 2004-10-10 & 2110 & - & - & - & 24.56 & -0.12 & 24.49 \\
\hline
\end{tabular}


H. Hildebrandt et al.: GaBoDS. V. ESO Deep Public Survey, Online Material p 9

Table A.6. Photometric solutions in the $I$-band. The colour term corresponds to the Johnson-Cousins colour $R-I$. The default value for the extinction coefficient in the one- and two-parameter fits is EXT1 $=$ EXT2 $=-0.10$ and the default value for the colour term in the one-parameter fit is $\mathrm{CT} 1=0.11$.

\begin{tabular}{|c|c|c|c|c|c|c|c|}
\hline Night & GaBoDS ID & $\mathrm{ZP} 3$ & EXT3 & CT3 & $\mathrm{ZP2}$ & CT2 & $\mathrm{ZP} 1$ \\
\hline 1999-11-04 & 308 & 23.16 & 0.00 & 0.28 & 23.27 & 0.28 & 23.37 \\
\hline 1999-11-07 & 311 & 26.18 & -2.61 & 0.27 & 23.31 & 0.27 & 23.40 \\
\hline 1999-11-08 & 312 & 23.24 & -0.00 & 0.24 & 23.36 & 0.24 & 23.44 \\
\hline $2000-02-26$ & 422 & 22.97 & 0.00 & 0.29 & 23.09 & 0.28 & 23.18 \\
\hline 2000-03-29 & 454 & 25.13 & -1.66 & 0.16 & 23.11 & 0.10 & 23.11 \\
\hline $2000-03-30$ & 455 & 23.59 & -0.50 & 0.28 & 23.12 & 0.27 & 23.21 \\
\hline $2000-03-31$ & 456 & 22.87 & 0.00 & 0.32 & 22.99 & 0.32 & 23.11 \\
\hline 2000-04-01 & 457 & 22.99 & -0.00 & 0.22 & 23.11 & 0.22 & 23.17 \\
\hline 2000-04-05 & 461 & 23.00 & 0.00 & 0.28 & 23.13 & 0.28 & 23.22 \\
\hline 2000-04-06 & 462 & 23.19 & -0.12 & 0.31 & 23.17 & 0.31 & 23.28 \\
\hline 2000-07-04 & 551 & 23.10 & -0.04 & 0.35 & 23.16 & 0.35 & 23.44 \\
\hline $2000-07-28$ & 575 & 23.04 & -0.00 & 0.23 & 23.16 & 0.23 & 23.24 \\
\hline $2000-07-31$ & 578 & 23.06 & 0.00 & 0.26 & 23.18 & 0.26 & 23.28 \\
\hline $2000-08-01$ & 579 & 23.08 & 0.00 & 0.28 & 23.21 & 0.28 & 23.32 \\
\hline $2000-08-03$ & 581 & 22.81 & 0.00 & 0.26 & 22.92 & 0.26 & 23.02 \\
\hline 2000-1 & 699 & 23.13 & -0.09 & 0.31 & 23.15 & 0.31 & 23.26 \\
\hline 2000-1 & 725 & 23.04 & 0.00 & 0.32 & 23.15 & 0.32 & 23.27 \\
\hline $2000-1$ & 726 & 23.04 & -0.01 & 0.31 & 23.17 & 0.30 & 23.28 \\
\hline 2001 & 783 & 23.07 & -0.18 & 0.28 & 22.97 & 0.29 & 23.06 \\
\hline 2001- & 814 & 23.09 & -0.11 & 0.29 & 23.08 & 0.28 & 23.18 \\
\hline 2001- & 815 & 22.95 & 0.00 & 0.29 & 23.08 & 0.29 & 23.18 \\
\hline 2001 & 901 & 23.15 & -0.09 & 0.29 & 23.17 & 0.29 & 23.28 \\
\hline $2001-06-20$ & 902 & 23.77 & 0.00 & -1.05 & 23.88 & -0.99 & 23.29 \\
\hline $2001-06-21$ & 903 & 23.18 & -0.09 & 0.29 & 23.20 & 0.29 & 23.30 \\
\hline $2001-06-22$ & 904 & 23.29 & 0.00 & -1.02 & 23.40 & -1.01 & 22.77 \\
\hline 2001-06-24 & 906 & 23.02 & 0.00 & 0.34 & 23.14 & 0.36 & 23.32 \\
\hline $2001-06-25$ & 907 & 23.10 & -0.08 & 0.37 & 23.13 & 0.37 & 23.31 \\
\hline 2001-06-26 & 908 & 23.04 & 0.00 & 0.27 & 23.17 & 0.29 & 23.27 \\
\hline 2001-06-29 & 911 & 23.21 & -0.14 & 0.38 & 23.16 & 0.37 & 23.46 \\
\hline 2001-06-30 & 912 & 23.05 & -0.08 & 0.43 & 23.08 & 0.43 & 23.39 \\
\hline $2001-07-20$ & 932 & 25.32 & -1.97 & 0.48 & 22.75 & 0.57 & 23.31 \\
\hline $2001-07-21$ & 933 & 22.59 & 0.00 & 0.60 & 22.73 & 0.59 & 23.27 \\
\hline $2001-07-22$ & 934 & 23.08 & 0.00 & 0.33 & 23.20 & 0.34 & 23.45 \\
\hline $2001-07-23$ & 935 & 23.64 & -0.50 & 0.35 & 23.11 & 0.35 & 23.41 \\
\hline 2001- & 936 & 23.07 & 0.00 & 0.34 & 23.19 & 0.34 & 23.44 \\
\hline 2001-0 & 937 & 23.07 & 0.00 & 0.36 & 23.21 & 0.36 & 23.52 \\
\hline $2001-0$ & 938 & 23.13 & -0.05 & 0.35 & 23.20 & 0.35 & 23.50 \\
\hline 2001-07- & 939 & 22.66 & 0.00 & 0.40 & 22.81 & 0.40 & 23.18 \\
\hline 2001-( & 962 & 23.08 & 0.00 & 0.25 & 23.21 & 0.25 & 23.29 \\
\hline 2001-08-21 & 964 & 23.00 & -0.02 & 0.41 & 23.10 & 0.42 & 23.41 \\
\hline
\end{tabular}

Table A.7. Photometric solutions in the $I_{-} E I S$-band. The colour term corresponds to the Johnson-Cousins colour $R-I$. The default value for the extinction coefficient in the one- and two-parameter fits is EXT1 $=$ EXT2 $=0.00$ and the default value for the colour term in the one-parameter fit is $\mathrm{CT} 1=0.03$.

\begin{tabular}{cr|rrr|rr|r}
\hline \hline Night & GaBoDS ID & ZP3 & EXT3 & CT3 & ZP2 & CT2 & ZP1 \\
\hline $2002-03-10$ & 1165 & 23.27 & 0.00 & 0.06 & 23.27 & 0.06 & 23.28 \\
$2002-03-11$ & 1166 & 23.33 & 0.00 & 0.06 & 23.33 & 0.06 & 23.35 \\
$2002-03-12$ & 1167 & 23.36 & 0.00 & 0.06 & 23.36 & 0.06 & 23.38 \\
$2002-03-13$ & 1168 & 23.39 & -0.02 & 0.05 & 23.37 & 0.06 & 23.38 \\
$2005-07-28$ & 2401 & 25.08 & -1.45 & 0.06 & 23.35 & 0.06 & 23.37 \\
\hline
\end{tabular}


Table B.1. Comparison of the GaBoDS (coaddition ID "D??A" and for the Deep2c $U$ - and $V$-band images "D2CB", respectively) and the EIS data. The mean and the standard deviation of the magnitude differences $\left(\Delta m=m_{\mathrm{GaBoDS}}-m_{\mathrm{EIS}}\right)$ are computed for objects in the magnitude range $17<m<21$. Due to the mistakes in the EIS photometric calibration (see text), in column four only the numbers printed in bold face are meaningful.

\begin{tabular}{|c|c|c|c|c|c|}
\hline Field & Filter & EIS ID & $\Delta m[\mathrm{mag}]$ & $\cos (\delta) \cdot \Delta \alpha\left[{ }^{\prime \prime}\right]$ & $\Delta \delta\left[{ }^{\prime \prime}\right]$ \\
\hline Deep1a & $\bar{U} U 35060$ & EIS.2004-11-05T11:29:36.834 & $-0.188 \pm \mathbf{0 . 0 4 0}$ & $0.045 \pm 0.117$ & $0.034 \pm 0.081$ \\
\hline Deep1a & $U \_38$ & EIS.2004-11-05T13:29:55.700 & $-0.172 \pm \mathbf{0 . 0 4 3}$ & $0.035 \pm 0.121$ & $0.043 \pm 0.079$ \\
\hline Deepla & $B^{-}$ & IS. 2004-11-04T20:33:40.955 & $-0.235 \pm \mathbf{0 . 0 4 8}$ & $-0.001 \pm 0.111$ & $.042 \pm 0.079$ \\
\hline Deepla & $R$ & IS. $2004-11-04 \mathrm{~T} 20: 41: 28.432$ & & & 079 \\
\hline Deepla & I & 02 & & 18 & .075 \\
\hline Deep1b & $U \_35060$ & IS. 200 & & $4 \pm 0$ & $5 \pm 0.085$ \\
\hline $\mathrm{p} 1 \mathrm{~b}$ & $B$ & IS. 200 & & $8 \pm 0.098$ & $6 \pm 0.069$ \\
\hline $\mathrm{p} 1 \mathrm{~b}$ & $V$ & IS. 200 & -0.0 & $0.035 \pm 0.091$ & -0.0 \\
\hline & $R$ & IS. 200 & $-0.124 \pm \mathbf{0}$. & $0.041 \pm 0.096$ & $7 \pm 0.060$ \\
\hline $1 b$ & I & IS. $2004-11-04 \mathrm{~T} 21: 55: 23.563$ & $-0.137 \pm \mathbf{0 . 1}$ & $0.047 \pm 0.087$ & $-0.018 \pm 0.058$ \\
\hline & $V$ & IS. $2004-11-04 \mathrm{~T} 20: 58: 46.283$ & $-0.027 \pm \mathbf{0 . 0 8 5}$ & $-0.015 \pm 0.153$ & $-0.035 \pm 0.070$ \\
\hline & $R$ & $-11-04 \mathrm{~T} 21: 45: 25.126$ & $-0.108 \pm \mathbf{0}$ & $3 \pm 0.146$ & $-0.043 \pm 0.071$ \\
\hline & $R$ & IS. 200 & -0.0 & $3 \pm 0.1$ & $8 \pm 0.132$ \\
\hline & $U_{-}$ & {$[S .200$} & & \pm 0 & \pm 0.107 \\
\hline & $B$ & IS. 200 & & 10 & $1 \pm 0.091$ \\
\hline & $V$ & IS. 200 & & & \pm 0.088 \\
\hline & $R$ & IS. 20 & & 21 & \pm 0.095 \\
\hline & I & IS. 20 & & 10 & 098 \\
\hline & & {$[S .2$} & & 16 & 096 \\
\hline & $V$ & {$[S .2$} & & 26 & 22 \\
\hline & $I$ & S. 2 & -0 . & \pm 0 . & 094 \\
\hline & $R$ & S. 20 & -0 & \pm 0 & \pm 0.075 \\
\hline & $U_{-}$ & [S.20 & $-0.200 \pm \mathbf{0 . 0 3 9}$ & $9 \pm 0.147$ & \pm 0.144 \\
\hline & $U_{-}$ & SS.200 & $16 \pm \mathbf{0 . 0 4 4}$ & $-0.208 \pm 0.162$ & $9 \pm 0.141$ \\
\hline & $B$ & $S .200$ & -0.1 & \pm 0.125 & \pm 0.130 \\
\hline & V & 5.20 & 0 & \pm 0 & \pm 0.129 \\
\hline & $R$ & S. 2 & -0.0 & 14 & .134 \\
\hline & & 5.20 & 71 & \pm 0 & \pm 0.079 \\
\hline & $B$ & 98 & -0.0 & 74 & \pm 0.060 \\
\hline & $V$ & 7 & & 971 & \pm 0.060 \\
\hline & $R$ & 26 & -0.0 & $6 \pm 0.076$ & $5 \pm 0.061$ \\
\hline & $I$ & 12 & & & $-0.337 \pm 0.054$ \\
\hline & & & & & \\
\hline & $B$ & & & & \\
\hline & $V$ & 17 & & 82 & \\
\hline & $R$ & IS. 200 & & & \\
\hline & $I$ & IS. $2004-11-05 ?$ & & & \\
\hline & $V$ & 49 & & & $-0.342 \pm 0.065$ \\
\hline & & EIS.2004-11-05T01:14:19.620 & $-0.104 \pm \mathbf{0 . 0 3 5}$ & $-0.363 \pm 0.064$ & $-0.353 \pm 0.056$ \\
\hline
\end{tabular}

Table C.1. FITS header keywords for the released images. The astrometric keywords from RADECSYS to CDELT2 are described in Greisen \& Calabretta (2002).

\begin{tabular}{lll}
\hline \hline Keyword & Unit & comment \\
\hline RADECSY & & astrometric system \\
CTYPE1 & & WCS projection type for $x$-axis \\
CUNIT1 & & $x$-axis unit \\
CRVAL1 & deg & world $x$-coordinate of reference pixel \\
CRPIX1 & pix & reference pixel on $x$-axis \\
CDELT1 & deg/pix & pixel step along $x$-axis \\
CTYPE2 & & WCS projection type for $y$-axis \\
CUNIT2 & & $y$-axis unit \\
CRVAL2 & deg & world $y$-coordinate of reference pixel \\
CRPIX2 & pix & reference pixel on $y$-axis \\
CDELT2 & deg/pix & pixel step along $y$-axis \\
EXPTIME & sec & total exposure time \\
GAIN & & effective GAIN (instrumental gain $[2.0] \times$ EXPTIME) \\
MAGZP & mag & Vega magnitude zeropoint \\
SEEING & arcsec & measured image seeing \\
COND1-5 & & condition on the input images entering coaddition \\
NIGHT1-3 & & GaBoDS IDs and solutions for nights included in phot. solution \\
\hline
\end{tabular}

